\title{
Impact of Channel Estimation-and-Artificial Noise Cancellation Imperfection on Artificial Noise-Aided Energy Harvesting Overlay Networks
}

\section{Khuong Ho-Van ( $\sim$ hvkhuong@hcmut.edu.vn )}

Ho Chi Minh City University of Technology https://orcid.org/0000-0001-7044-4131

\section{Thiem Do-Dac}

Thu Dau Mot University

\section{Research Article}

Keywords: Overlay, secrecy outage probability, energy harvesting, channel estimation imperfection, artificial noise cancellation.

Posted Date: February 25th, 2021

DOI: https://doi.org/10.21203/rs.3.rs-176103/v1

License: (c) (i) This work is licensed under a Creative Commons Attribution 4.0 International License.

Read Full License

Version of Record: A version of this preprint was published at Telecommunication Systems on July 5th, 2021. See the published version at https://doi.org/10.1007/s11235-021-00808-8. 


\title{
Impact of Channel Estimation-and-Artificial
}

\section{Noise Cancellation Imperfection on Artificial Noise-Aided Energy Harvesting Overlay Networks}

\author{
K. Ho-Van and T. Do-Dac
}

\begin{abstract}
EHONs (Energy Harvesting Overlay Networks) satisfy stringent design requirements such as high energy-and-spectrum utilization efficiencies. However, due to open access nature of these networks, eavesdroppers can emulate cognitive radios to wire-tap legitimate information, inducing information security to become a great concern. In order to protect legitimate information against eavesdroppers, this paper generates artificial noise transmitted simultaneously with legitimate information to interfere eavesdroppers. Nonetheless, artificial noise cannot be perfectly suppressed at legitimate receivers as for its primary purpose of interfering only eavesdroppers. Moreover, channel information used for signal detection is hardly estimated at receivers with absolute accuracy. As such, to quickly evaluate impact of channel estimation-and-artificial noise cancellation imperfection on secrecy performance of secondary/primary communication in ANaEHONs (Artificial Noise-aided EHONs), this paper firstly proposes precise closed-form formulas of primary/secondary SOP (Secrecy Outage Probability). Then, computer simulations are provided to corroborate these formulas. Finally, various results are illustrated to shed insights into secrecy performance of ANaEHON with key system parameters from which optimum parameters are recognized. Notably, secondary/primary communication can be secured at different levels by flexibly adjusting various parameters of the proposed system model.
\end{abstract}

\section{Index Terms}

Overlay; secrecy outage probability; energy harvesting; channel estimation imperfection; artificial noise cancellation.

K. Ho-Van and T. Do-Dac are with HCMUT, VN (e-mail: hvkhuong@hcmut.edu.vn); Thiem Do-Dac is also with TDMU, VN (dodacthiem@gmail.com). 


\section{INTRODUCTION}

Advanced wireless networks such as 5G/6G (Fifth/Sixth Generation) open a door to a large number of emerging wireless applications but impose an immense pressure on telecommunications infrastructure which requires advanced technology solutions of high (spectrum utilization, energy, spectral) efficiencies to release it [1]-[3]. Indeed, a key application of 5G networks is IoT (Internet of Things), which is deployed extensively from civilian (e.g., transportation, electricity, healthcare, public safety, ... ) to military (e.g., tactical reconnaissance, smart bases, ...) [4]. However, when deploying IoT, an enormous number of concurrently connected terminals consume tremendous amount of energy and hence, it is essential to improve energy efficiency to not only extend the lifetime of terminals but also reduce energy need. Moreover, IoT demands a large bandwidth to allot concurrently a huge number of terminals and thus, in the spectrum shortage-and-scarcity situation as nowadays, solutions of enhancing spectral efficiency should be devised. Similarly to IoT, 5G mobile wireless communications, which serves the growing number of mobile terminals and demands increasingly high data transmission speed, needs efficient energy-and-spectrum utilization solutions to meet its requirements [5].

CRs (Cognitive Radios), which typically operate in overlay, underlay, and interweave modes, can access the licensed frequency band of PUs (Primary Users) without causing any performance degradation for PUs, thus significantly improving spectral efficiency and mitigating spectrum scarcity issue [6]. In the underlay mode, CRs utilize the licensed spectrum but must upperbound interference caused at PUs. The overlay mode allows concurrent transmission of CRs and PUs but signal reception quality at primary receivers must be remained or enhanced with complicated signal processing techniques. In the meantime, the interweave mode merely leaves blank licensed spectrum for CRs to utilize. While literature has intensively focused on the underlay and interweave modes, few works have studied the overlay one. The overlay mode can trade-off performances between primary and secondary communication better than other modes and hence, it is of a special attention in the current paper.

Energy efficiency of wireless communication can be enhanced by several viable solutions (e.g., network planning, EH (Energy Harvesting), hardware solutions) amongst which, RF (Radio Frequency) energy harvesting neither requires additional energy scavenging equipments (e.g., solar panels, wind turbines) nor depends time-variant energy resources. Such advantages of this energy harvesting solution enable it to be integrated into (5G/6G mobile or IoT) users to supply 
energy, extend the life-time of wireless devices, and improve energy efficiency [7]. Relaying communication [8]-[10] or simultaneous wireless information and power transfer [11]-[13] is currently a means to implement this solution.

EHONs can exploit simultaneously advantages of both feasible (cognitive radio and energy harvesting) technologies to meet several standards of advanced wireless networks requiring high energy-and-spectral efficiencies [14]. Nevertheless, that both licensed and unlicensed users in these networks are permitted to utilize the licensed spectrum simultaneously may enable eavesdroppers to emulate legitimate users to steal secret information, seriously warning security issues. To supplement and improve secrecy capability for traditional cryptographic and encryption techniques, PLS (physical layer security) has recently been suggested [15]. Amongst various PLS methods (e.g., opportunistic scheduling, transmit beam-forming, transmit antenna selection, on-off transmission, jamming, relaying), jamming (or generating artificial noise) is of a great concern due to its simple, efficient, and flexible implementation [16]. Therefore, this paper applies artificial noise in EHONs to secure primary/secondary communication.

Most references (e.g., [17]-[21]) assumed artificial noise to be exactly known at legitimate receivers. Accordingly, these receivers completely eliminate its detrimental effect while eavesdroppers suffer severely this effect. Nevertheless, the amount of artificial noise received at legitimate receivers is variable due to uncertainties such as noise and fading. As such, assumption on perfect artificial noise cancellation at these receivers seems unrealistic. Moreover, channel information affects successful probability of signal detection not only at legitimate receivers but also eavesdroppers, eventually impact security capability. Nonetheless, it is certain that any channel estimator has some accuracy degree [22] and hence, it is practical to investigate channel estimation imperfection in ANaEHON. Therefore, this paper evaluates effect of channel estimation-and-artificial noise cancellation imperfection on security performance of PUs/CRs in ANaEHON.

\section{A. Prior works}

This paper considers $\mathrm{ANaEHON}$ where a primary transmitter-receiver pair cannot communicate with each other directly due to some reasons and a secondary transmitter-receiver pair assists primary communication in reward for their access to primary spectrum. The secondary transmitter harvests RF energy from the primary transmitter and transmits not only its private 
signal but also the primary transmitter's signal and artificial noise. Information transmission of the secondary transmitter is wire-tapped by an eavesdropper.

While publications on information security for energy harvesting (interweave/underlay) networks have been blooming, few works have been interested in the overlay mode [18]-[21], [23]-[25]. More specifically, [18] and [19] considered the almost same system model as ours but EHONs are secured by letting the primary receiver jam the eavesdropper and the secondary transmitter helps primary communication by the AF (Amplify-and-Forward) mechanism ${ }^{1}$. In [20], a dedicated jammer was employed to interfere the eavesdropper instead of the primary receiver as [18] and [19]. In addition, [20] differs [18] and [19] in the EH method, the EH-capable terminal, and the assistance mechanism. The former used the EH-capable jammer, which harvests energy based on the time splitting technique [27], and employed the secondary transmitter as a DF (Decode-and-Forward) relay. Meanwhile, the latter used the secondary transmitter as the $\mathrm{AF}$ relay and as an energy harvester which is based on the power splitting technique [28]. To further secure primary transmission, [21] proposed to jam the eavesdropper by both the primary receiver and the dedicated jammer. However, security performance of primary/secondary communication in terms of SOP was not analyzed in [18]-[21]. In [23], the transmit antenna selection and the multi-user scheduling were proposed to secure EHONs and the ergodic rate of secondary communication and the SOP of primary communication were derived in closed-form. Nonetheless, different from [18]-[21], the secondary user relays the primary signal and transmits its private signal separately in [23]. This significantly mitigates complexity in analyzing the SOP and hence, making the analysis in [23] tractable.

Although [23] analyzed the ergodic rate of secondary communication and the SOP of primary communication in EHONs, the SU (Secondary User) relays the primary signal and transmits its private signal separately. This requires at least three stages (Stage I: energy harvesting and PU's transmission, Stage II: SU's transmission to PU, Stage III: SU's transmission to SU) to complete a transmission process of both SU and PU, considerably reducing spectral efficiency. Recently, [24] and [25] proposed a two-stage transmission scheme with artificial noise generation in EHONs to improve spectral efficiency and secrecy performance. More specifically, [24] and [25] proposed the secondary transmitter to play dual role as the traditional secondary transmitter

\footnotetext{
${ }^{1}$ The system model in [18] and [19] was studied in [26]. However, the secondary transmitter was assumed to scavenge energy from the ambient rather than RF signals, considerably simplifying the analysis. Furthermore, [26] did not employ artificial noise. Therefore, references like [26] are not objectives to be surveyed.
} 
operating in the overlay mode (i.e., relay primary message and transmit secondary message) and the jammer by network-coding three (primary, secondary, artificial noise) signals. Moreover, [24] and [25] proposed the SOP analysis.

\section{B. Motivations and Contributions}

Imperfect channel estimation and artificial noise cancellation are ineluctable in practical systems and hence, this paper studies their impact on security performance of ANaEHON in [24] and [25]. It contributes the following:

- Propose a novel operation mechanism of the secondary transmitter which enables it to harvest energy from the primary transmitter, decode and forward primary information, and generate a signal combination of primary information, secondary information, and artificial noise. This mechanism is flexible in compromising security performance of primary communication with that of secondary communication and optimizing system design by selecting appropriately the (power splitting, time splitting, power allocation) factors.

- Propose precise closed-form SOP formulas for promptly assessing security performance of secondary/primary communication under channel estimation-and-artificial noise cancellation imperfection. These formulas serve as a key starting point to obtain formulas for other pivotal secrecy performance indicators comprising IP (Intercept Probability), STP (Secrecy Throughput), PSCP (Positive Secrecy Capacity Probability).

- Search optimum pivotal specifications for the best secrecy performance and the best performance trade-off between secondary and primary communication.

- Provide insightful results on security performance of primary/secondary communication in important system parameters.

\section{Structure}

Part II describes the system model. Next, Part III derives detailedly the SOP of primary/secondary communication. Subsequently, Part IV provides illustrative results and finally, Part V closes the paper.

\section{System Model}

Figure 1 shows an $\mathrm{ANaEHON}$ in which direct communication between a primary transmitterreceiver pair, $P T-P R$, is not of good quality owing to uncertainties (e.g., long distance, severe 
TABLE I

SUMmARY OF SYMBOLS

\begin{tabular}{|c|c|}
\hline Symbol & Meaning \\
\hline$x_{p}$ & Transmit symbol of $P T$ \\
\hline$x_{s}$ & Transmit symbol of $S T$ \\
\hline$x_{a}$ & Artificial noise \\
\hline$P_{p}$ & Transmit power of $P T$ \\
\hline$P_{s}$ & Transmit power of $S T$ \\
\hline$n_{s}$ & Noise at $S T$ \\
\hline$\tilde{n}_{s}$ & Noise due to the passband-to-baseband signal conversion at $S T$ \\
\hline$n_{e}$ & Noise at $E$ \\
\hline$n_{r}$ & Noise at $S R$ \\
\hline$n_{p}$ & Noise at $P R$ \\
\hline$h_{p s}$ & $P T-S T$ channel coefficient \\
\hline$h_{s p}$ & $S T-P R$ channel coefficient \\
\hline$h_{s e}$ & $S T-E$ channel coefficient \\
\hline$h_{s r}$ & $S T-S R$ channel coefficient \\
\hline$\mu_{p s}$ & Fading power of $P T-S T$ channel \\
\hline$\mu_{s p}$ & Fading power of $S T-P R$ channel \\
\hline$\mu_{s e}$ & Fading power of $S T-E$ channel \\
\hline$\mu_{s r}$ & Fading power of $S T-S R$ channel \\
\hline$y_{s}$ & Received signal at $S T$ \\
\hline$y_{e}$ & Received signal at $E$ \\
\hline$y_{r}$ & Received signal at $S R$ \\
\hline$y_{p}$ & Received signal at $P R$ \\
\hline$T$ & Total transmission time \\
\hline$\alpha$ & Time splitting factor \\
\hline$\lambda$ & Power splitting factor \\
\hline$\theta$ & Power allocation factor for desired signals and artificial noise when $S T$ decodes successfully $P T$ 's signal \\
\hline$\tau$ & Power allocation factor for artificial noise and secondary signal as $S T$ decodes incorrectly $P T$ 's signal \\
\hline$\kappa$ & Power allocation factor for primary and secondary signals \\
\hline$C_{t}$ & Transmission rate required by $S T$ \\
\hline$C_{0}$ & Required secrecy capacity \\
\hline$\gamma_{s}$ & SNR at $S T$ \\
\hline$\gamma_{e}$ & SINR at $E$ \\
\hline$\gamma_{r}$ & SINR at $S R$ \\
\hline$\gamma_{p}$ & SINR at $P R$ \\
\hline $\operatorname{Pr}\{X\}$ & Probability of the event $X$ \\
\hline$X \sim \mathcal{C N}(0, m)$ & Zero-mean $m$-variance circular symmetric complex Gaussian random variable \\
\hline$\varsigma$ & Path-loss exponent \\
\hline$d_{\mathrm{uv}}$ & Distance between a corresponding transmitter-receiver pair \\
\hline$\rho_{\mathrm{uv}}$ & Correlation coefficient between true and estimated channels \\
\hline$\chi$ & Artificial noise residue level \\
\hline
\end{tabular}



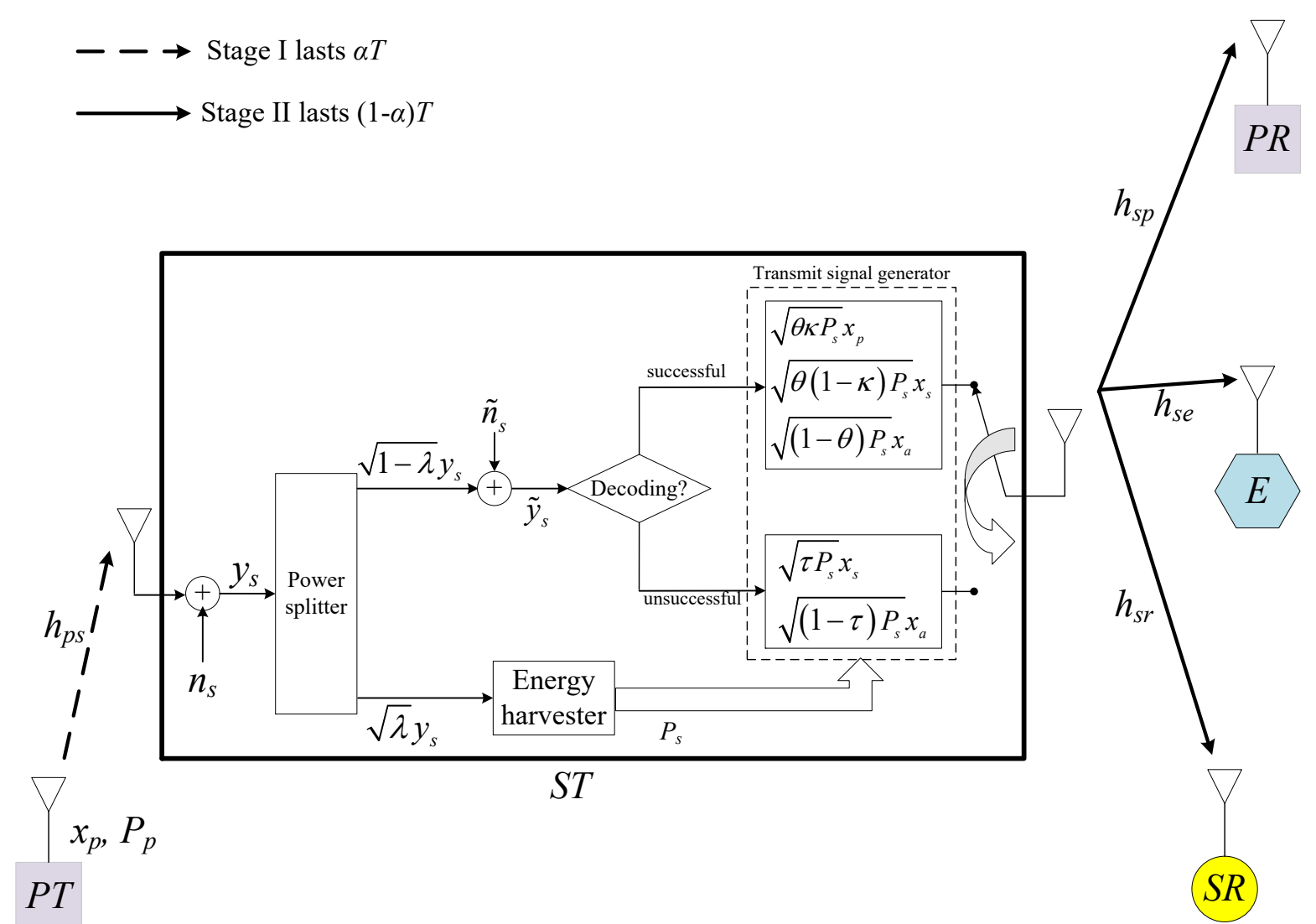

Fig. 1. System model.

fading, ...). Therefore, the secondary transmitter $S T$, which is in the transmission range of $P T$, can assist $P T$ in relaying the $P T$ 's signal to $P R$. ST is assumed to be capable of harvesting RF energy from $P T$ and consumes the scavenged energy for its communication operation. Additionally, $S T$ operates in the overlay mechanism where it not only relays the $P T$ 's signal to $P R$ but also sends its private signal to the secondary receiver $S R$. Information transmission of $S T$ is stolen by an eavesdropper $E$. In order to reduce the wire-tapping capability of $E, S T$ transmits artificial noise together with information signals of PT and ST.

Table I summarizes main notations used throughout this paper. More specifically, as shown in Figure $1, h_{p s}, h_{s p}, h_{s e}$, and $h_{s r}$ correspondingly signify channel coefficients between $P T$ and $S T, S T$ and $P R, S T$ and $E, S T$ and $S R$. In the current paper, these channel coefficients are modelled as $h_{p s} \sim \mathcal{C N}\left(0, \mu_{p s}\right), h_{s p} \sim \mathcal{C N}\left(0, \mu_{s p}\right), h_{s e} \sim \mathcal{C N}\left(0, \mu_{s e}\right)$, and $h_{s r} \sim \mathcal{C N}\left(0, \mu_{s r}\right)$, respectively. Such a channel model indicates Rayleigh fading. Path-loss can be incorporated into 
$\mu_{\mathrm{uv}}$ with $\mathrm{u} \in\{p, s\}$ and $\mathrm{v} \in\{s, p, r, e\}$ as $\mu_{\mathrm{uv}}=d_{\mathrm{uv}}^{-\varsigma}$ in which $\varsigma$ is the path-loss exponent and $d_{\mathrm{uv}}$ is the $\mathrm{u}-\mathrm{v}$ distance. Then, the probability density function (PDF) and the cumulative distribution function (CDF) of $\left|h_{\mathrm{uv}}\right|^{2}$ are correspondingly expressed as $f_{\left|h_{\mathrm{uv}}\right|^{2}}(x)=e^{-x / \mu_{\mathrm{uv}}} / \mu_{\mathrm{uv}}$ and $F_{\left|h_{\mathrm{uv}}\right|^{2}}(x)=1-e^{-x / \mu_{\mathrm{uv}}}$, where $x \geq 0$.

In Figure 1, the total transmission time $T$ for both $P T$ and $S T$ to complete their information transmission to corresponding receivers is divided into two stages. Stage I with the time of $\alpha T$ with $\alpha \in(0,1)$ being the time splitting factor is for $P T$ to transmit its unit-power symbol $x_{p}$ with the transmit power of $P_{p}$ in order for $S T$ to harvest energy based on the power splitting technique and decode the $P T$ 's information. This technique separates the received signal of $S T, y_{s}$, into two portions: one portion $\sqrt{\lambda} y_{s}$ with $\lambda \in(0,1)$ being the power splitting factor for decoding the PT's information ${ }^{2}$ and another portion $\sqrt{1-\lambda} y_{s}$ for harvesting energy. Dependent on the decoding status $^{3}, S T$ transmits distinct signals. To be specific, if $S T$ successfully restores the $P T$ 's information, it sends a combination of three signals $\sqrt{\theta \kappa P_{s}} x_{p}+\sqrt{\theta(1-\kappa) P_{s}} x_{s}+\sqrt{(1-\theta) P_{s}} x_{a}$ $\left(P_{s}, \theta\right.$ and $\kappa$ are the transmit power of $S T$, the power allocation factor for desired signals and artificial noise as $S T$ decodes correctly the $P T$ 's signal and the power allocation factor for primary and secondary signals, respectively): the $P T$ 's decoded information $x_{p}$, the $S T$ 's private information $x_{s}$, and the artificial noise $x_{a}$. In the case that $S T$ unsuccessfully decodes the PT's information, it transmits a superposition of two signals $\sqrt{\tau P_{s}} x_{s}+\sqrt{(1-\tau) P_{s}} x_{a}(\tau$ is the power allocation factor for desired signal and artificial noise as $S T$ decodes incorrectly the PT's signal): the ST's private information $x_{s}$ and the artificial noise $x_{a}$. Stage II with the time of $(1-\alpha) T$ is for $S T$ to send its signal to $S R, P R$, and $E$.

In Stage I, $S T$ receives the following signal

$$
y_{s}=h_{p s} \sqrt{P_{p}} x_{p}+n_{s}
$$

where the receive antenna at $S T$ induces the noise $n_{s} \sim \mathcal{C N}\left(0, \sigma_{s}^{2}\right)$.

According to Figure 1, ST scavenges the total energy in Stage I as

$$
E_{s}=\eta \Xi\left\{\left|\sqrt{\lambda} y_{s}\right|^{2}\right\} \alpha T=\alpha \eta \lambda\left(P_{p} \mu_{p s}+\sigma_{s}^{2}\right) T,
$$

\footnotetext{
${ }^{2}$ The information decoder assumably consumes negligible energy, which is widely acknowledged in previous publications (e.g., [20], [29]-[34]).

${ }^{3}$ In [20], $S T$ always relays $P T$ 's message in Stage II. This can cause error propagation for PT's message. However, [20] did not analyze the SOP of primary/secondary communication and hence, error propagation was not accounted for the SOP analysis.
} 
where $\Xi\{\cdot\}$ is the expectation operator and $\eta \in(0,1)$ is the energy conversion efficiency.

The power which $S T$ can consume in Stage II is

$$
P_{s}=\frac{E_{s}}{(1-\alpha) T}=\frac{\alpha \eta \lambda}{1-\alpha}\left(P_{p} \mu_{p s}+\sigma_{s}^{2}\right) .
$$

Figure 1 shows that the signal used for decoding the $P T$ 's information is

$$
\tilde{y}_{s}=\sqrt{1-\lambda} y_{s}+\tilde{n}_{s}
$$

where the passband-to-baseband signal conversion induces the noise $\tilde{n}_{s} \sim \mathcal{C N}\left(0, \tilde{\sigma}_{s}^{2}\right)$.

Plugging (1) into (4) results in

$$
\tilde{y}_{s}=\sqrt{(1-\lambda) P_{p}} h_{p s} x_{p}+\sqrt{1-\lambda} n_{s}+\tilde{n}_{s} .
$$

Channel estimators suffer a certain error and hence, channel state information is not perfectly estimated. For performance analysis, channel estimation imperfection should be modelled appropriately. This paper employs a well-known channel estimation error model as [22]

$$
\tilde{h}_{\mathrm{uv}}=\rho_{\mathrm{uv}} h_{\mathrm{uv}}+\sqrt{1-\rho_{\mathrm{uv}}^{2}} \varepsilon_{\mathrm{uv}}
$$

where $h_{\mathrm{uv}}$ is the true channel, $\tilde{h}_{\mathrm{uv}}$ is the estimated channel, $\varepsilon_{\mathrm{uv}}$ is the estimation error; all random variables $h_{\mathrm{uv}}, \tilde{h}_{\mathrm{uv}}, \varepsilon_{\mathrm{uv}}$ are modelled as $\mathcal{C N}\left(0, \mu_{\mathrm{uv}}\right)$; the correlation coefficient $0 \leq \rho_{\mathrm{uv}} \leq 1$ is a constant, representing the exactness of channel estimation.

Inserting (6) into (5), one obtains

$$
\begin{aligned}
\tilde{y}_{s} & =\sqrt{(1-\lambda) P_{p}}\left(\frac{\tilde{h}_{p s}}{\rho_{p s}}-\frac{\sqrt{1-\rho_{p s}^{2}}}{\rho_{p s}} \varepsilon_{p s}\right) x_{p}+\sqrt{1-\lambda} n_{s}+\tilde{n}_{s} \\
& =\frac{\sqrt{(1-\lambda) P_{p}}}{\rho_{p s}} \tilde{h}_{p s} x_{p}-\frac{\sqrt{(1-\lambda) P_{p}\left(1-\rho_{p s}^{2}\right)}}{\rho_{p s}} \varepsilon_{p s} x_{p}+\sqrt{1-\lambda} n_{s}+\tilde{n}_{s} .
\end{aligned}
$$

It is inferred from (7) that the SNR (Signal-to-Noise Ratio) achievable for decoding the PT's information is given by

$$
\gamma_{s}=\frac{\Xi\left\{\left|\frac{\sqrt{(1-\lambda) P_{p}}}{\rho_{p s}} \tilde{h}_{p s} x_{p}\right|^{2}\right\}}{\Xi\left\{\left|-\frac{\sqrt{(1-\lambda) P_{p}\left(1-\rho_{p s}^{2}\right)}}{\rho_{p s}} \varepsilon_{p s} x_{p}+\sqrt{1-\lambda} n_{s}+\tilde{n}_{s}\right|^{2}\right\}}=D\left|\tilde{h}_{p s}\right|^{2},
$$

where

$$
D=\frac{P_{p}}{P_{p}\left(1-\rho_{p s}^{2}\right) \mu_{p s}+\left(\sigma_{s}^{2}+\frac{\tilde{\sigma}_{s}^{2}}{1-\lambda}\right) \rho_{p s}^{2}} .
$$


The channel capacity that $S T$ can obtain is $C_{s}=\alpha \log _{2}\left(1+\gamma_{s}\right)$ bps/Hz with the pre-logarithm factor $\alpha$ owing to Stage I of $\alpha T$. The communication theory addressed that $S T$ decodes exactly the PT's information merely if its channel capacity is larger than the required transmission rate $C_{t}$, i.e., $C_{s} \geq C_{t}$. In other words, $x_{p}$ is decoded accurately at $S T$ if $\gamma_{s} \geq \gamma_{t}$ where $\gamma_{t}=2^{C_{t} / \alpha}-1$.

If $S T$ decodes successfully the PT's information, it broadcasts the combination of three signals in the form of $\sqrt{\theta \kappa P_{s}} x_{p}+\sqrt{\theta(1-\kappa) P_{s}} x_{s}+\sqrt{(1-\theta) P_{s}} x_{a}$ in Stage II. Otherwise, it broadcasts the combination of solely two signals in the form of $\sqrt{\tau P_{s}} x_{s}+\sqrt{(1-\tau) P_{s}} x_{a}$ in Stage II. Therefore, $P R, S R$, and $E$ receive signals in Stage II, correspondingly, as

$$
\begin{gathered}
y_{p}=\left\{\begin{array}{cc}
h_{s p}\left(\sqrt{\theta \kappa P_{s}} x_{p}+\sqrt{\theta(1-\kappa) P_{s}} x_{s}+\sqrt{(1-\theta) P_{s}} x_{a}\right)+n_{p} & , \gamma_{s} \geq \gamma_{t} \\
h_{s p}\left(\sqrt{\tau P_{s}} x_{s}+\sqrt{(1-\tau) P_{s}} x_{a}\right)+n_{p} & , \gamma_{s}<\gamma_{t}
\end{array}\right. \\
y_{r}=\left\{\begin{array}{cc}
h_{s r}\left(\sqrt{\theta \kappa P_{s}} x_{p}+\sqrt{\theta(1-\kappa) P_{s}} x_{s}+\sqrt{(1-\theta) P_{s}} x_{a}\right)+n_{r} & , \gamma_{s} \geq \gamma_{t} \\
h_{s r}\left(\sqrt{\tau P_{s}} x_{s}+\sqrt{(1-\tau) P_{s}} x_{a}\right)+n_{r} & , \gamma_{s}<\gamma_{t}
\end{array}\right. \\
y_{e}=\left\{\begin{array}{cc}
h_{s e}\left(\sqrt{\theta \kappa P_{s}} x_{p}+\sqrt{\theta(1-\kappa) P_{s}} x_{s}+\sqrt{(1-\theta) P_{s}} x_{a}\right)+n_{e} & , \gamma_{s} \geq \gamma_{t} \\
h_{s e}\left(\sqrt{\tau P_{s}} x_{s}+\sqrt{(1-\tau) P_{s}} x_{a}\right)+n_{e} & , \gamma_{s}<\gamma_{t}
\end{array}\right.
\end{gathered}
$$

where $n_{p} \sim \mathcal{C N}\left(0, \sigma_{p}^{2}\right), n_{r} \sim \mathcal{C N}\left(0, \sigma_{r}^{2}\right), n_{e} \sim \mathcal{C N}\left(0, \sigma_{e}^{2}\right)$ are respectively the noises impaired by the receive antennas at $P R, S R$, and $E$.

Most works (e.g., [17]-[21]) assumed the artificial noise $x_{a}$ to be completely known at the legitimate receivers $(P R$ and $S R$ ), not at $E$, in order for $P R$ and $S R$ to totally eliminate the effect of $x_{a}$ on their received signal but this assumption seems impractical because the regeneration of $x_{a}$ is hardly achieved with the absolute probability. Therefore, this paper assumes $x_{a}$ to be regenerated at $P R$ and $S R$ with the accuracy of $1-\chi, \chi \in[0,1]$, which indicates that $\chi x_{a}$ represents the residual artificial noise due to imperfect artificial noise cancellation at $P R$ and $S R$. Accordingly, $P R$ and $S R$ obtain signals with less artificial noise after partly removing $x_{a}$, respectively, as

$$
\begin{aligned}
& \tilde{y}_{p}=\left\{\begin{array}{cc}
h_{s p}\left(\sqrt{\theta \kappa P_{s}} x_{p}+\sqrt{\theta(1-\kappa) P_{s}} x_{s}+\chi \sqrt{(1-\theta) P_{s}} x_{a}\right)+n_{p} & , \gamma_{s} \geq \gamma_{t} \\
h_{s p}\left(\sqrt{\tau P_{s}} x_{s}+\chi \sqrt{(1-\tau) P_{s}} x_{a}\right)+n_{p} & , \gamma_{s}<\gamma_{t}
\end{array}\right. \\
& \tilde{y}_{r}=\left\{\begin{array}{cc}
h_{s r}\left(\sqrt{\theta \kappa P_{s}} x_{p}+\sqrt{\theta(1-\kappa) P_{s}} x_{s}+\chi \sqrt{(1-\theta) P_{s}} x_{a}\right)+n_{r} & , \gamma_{s} \geq \gamma_{t} \\
h_{s r}\left(\sqrt{\tau P_{s}} x_{s}+\chi \sqrt{(1-\tau) P_{s}} x_{a}\right)+n_{r} & , \gamma_{s}<\gamma_{t}
\end{array}\right.
\end{aligned}
$$


Inserting (6) into (13), one obtains

$$
\begin{gathered}
\tilde{y}_{p}=\left\{\begin{array}{cl}
\left(\frac{\tilde{h}_{s p}}{\rho_{s p}}-\frac{\sqrt{1-\rho_{s p}^{2}}}{\rho_{s p}} \varepsilon_{s p}\right)\left(\sqrt{\theta \kappa P_{s}} x_{p}+\sqrt{\theta(1-\kappa) P_{s}} x_{s}+\chi \sqrt{(1-\theta) P_{s}} x_{a}\right)+n_{p} & , \gamma_{s} \geq \gamma_{t} \\
h_{s p}\left(\sqrt{\tau P_{s}} x_{s}+\chi \sqrt{(1-\tau) P_{s}} x_{a}\right)+n_{p} & , \gamma_{s}<\gamma_{t}
\end{array}\right. \\
=\left\{\begin{array}{cl}
\frac{\sqrt{\theta \kappa P_{s}}}{\rho_{s p}} \tilde{h}_{s p} x_{p}-\frac{\sqrt{\theta \kappa\left(1-\rho_{s p}^{2}\right) P_{s}}}{\rho_{s p}} \varepsilon_{s p} x_{p}+\left(\frac{\tilde{h}_{s p}}{\rho_{s p}}-\frac{\sqrt{1-\rho_{s p}^{2}}}{\rho_{s p}} \varepsilon_{s p}\right)\left(\sqrt{\theta(1-\kappa) P_{s}} x_{s}+\chi \sqrt{(1-\theta) P_{s}} x_{a}\right)+n_{p}, \gamma_{s} \geq \gamma_{t} \\
h_{s p}\left(\sqrt{\tau P_{s}} x_{s}+\chi \sqrt{(1-\tau) P_{s}} x_{a}\right)+n_{p} & , \gamma_{s}<\gamma_{t}
\end{array}\right.
\end{gathered}
$$

Based on (17), SINR (Signal-to-Interference plus Noise Ratio) for decoding $x_{p}$ at $P R$ is expressed as

$$
\begin{aligned}
& \gamma_{p}=\left\{\begin{array}{cl}
\Xi\left\{\left|\frac{\sqrt{\theta \kappa P_{s}}}{\rho_{s p}} \tilde{h}_{s p} x_{p}\right|^{2}\right\} & , \gamma_{s} \geq \gamma_{t} \\
\Xi\left\{\left|-\frac{\sqrt{\theta \kappa\left(1-\rho_{s p}^{2}\right) P_{s}}}{\rho_{s p}} \varepsilon_{s p} x_{p}+\left(\frac{\tilde{h}_{s p}}{\rho_{s p}}-\frac{\sqrt{1-\rho_{s p}^{2}}}{\rho_{s p}} \varepsilon_{s p}\right)\left(\sqrt{\theta(1-\kappa) P_{s}} x_{s}+\chi \sqrt{(1-\theta) P_{s}} x_{a}\right)+n_{p}\right|^{2}\right\} & \\
0 & , \gamma_{s}<\gamma_{t}
\end{array}\right. \\
& =\left\{\begin{array}{cl}
\frac{\theta \kappa P_{s}\left|\tilde{h}_{s p}\right|^{2}}{\left[\theta(1-\kappa)+\chi^{2}(1-\theta)\right] P_{s}\left|\tilde{h}_{s p}\right|^{2}+\left[\theta+\chi^{2}(1-\theta)\right]\left(1-\rho_{s p}^{2}\right) \mu_{s p} P_{s}+\rho_{s p}^{2} \sigma_{p}^{2}} & , \gamma_{s} \geq \gamma_{t} \\
0 & , \gamma_{s}<\gamma_{t}
\end{array}\right.
\end{aligned}
$$

Similarly, inserting (6) into (14), one obtains

$$
\begin{gathered}
\tilde{y}_{r}=\left\{\begin{array}{cc}
\left(\frac{\tilde{h}_{s r}}{\rho_{s r}}-\frac{\sqrt{1-\rho_{s r}^{2}}}{\rho_{s r}} \varepsilon_{s r}\right)\left(\sqrt{\theta \kappa P_{s}} x_{p}+\sqrt{\theta(1-\kappa) P_{s}} x_{s}+\chi \sqrt{(1-\theta) P_{s}} x_{a}\right)+n_{r} & , \gamma_{s} \geq \gamma_{t} \\
\left(\frac{\tilde{h}_{s r}}{\rho_{s r}}-\frac{\sqrt{1-\rho_{s r}^{2}}}{\rho_{s r}} \varepsilon_{s r}\right)\left(\sqrt{\tau P_{s}} x_{s}+\chi \sqrt{(1-\tau) P_{s}} x_{a}\right)+n_{r} & , \gamma_{s}<\gamma_{t}
\end{array}\right. \\
=\left\{\begin{array}{c}
\sqrt{\theta(1-\kappa) P_{s} \frac{\tilde{h}_{s r}}{\rho_{s r}} x_{s}-\frac{\sqrt{\left(1-\rho_{s r}^{2}\right) \theta(1-\kappa) P_{s}}}{\rho_{s r}}} \varepsilon_{s r} x_{s}+\left(\frac{\tilde{h}_{s r}}{\rho_{s r}}-\frac{\sqrt{1-\rho_{s r}^{2}}}{\rho_{s r}} \varepsilon_{s r}\right)\left(\sqrt{\theta \kappa P_{s}} x_{p}+\chi \sqrt{(1-\theta) P_{s}} x_{a}\right)+n_{r}, \gamma_{s} \geq \gamma_{t} \\
\frac{\tilde{h}_{s r}}{\rho_{s r}} \sqrt{\tau P_{s}} x_{s}-\frac{\sqrt{\left(1-\rho_{s r}^{2}\right) \tau P_{s}}}{\rho_{s r}} \varepsilon_{s r} x_{s}+\left(\frac{\tilde{h}_{s r}}{\rho_{s r}}-\frac{\sqrt{1-\rho_{s r}^{2}}}{\rho_{s r}} \varepsilon_{s r}\right) \chi \sqrt{(1-\tau) P_{s}} x_{a}+n_{r} \quad, \gamma_{s}<\gamma_{t}
\end{array}\right.
\end{gathered}
$$


Based on (17), SINR for decoding $x_{s}$ at $S R$ is given by

$$
\begin{aligned}
& \gamma_{r}=\left\{\begin{array}{cc}
\Xi\left\{\left|\sqrt{\theta(1-\kappa) P_{s}} \frac{\tilde{h}_{s r} \rho_{s r}}{\rho_{s}}\right|^{2}\right\} & c_{\Xi\left\{-\frac{\sqrt{\left(1-\rho_{s r}^{2}\right) \theta(1-\kappa) P_{s}}}{\rho_{s r}} \varepsilon_{s r} x_{s}+\left(\frac{\tilde{h}_{s r}}{\rho_{s r}}-\frac{\sqrt{1-\rho_{s r}^{2}}}{\rho_{s r}} \varepsilon_{s r}\right)\left(\sqrt{\theta \kappa P_{s}} x_{p}+\chi \sqrt{(1-\theta) P_{s}} x_{a}\right)+\left.n_{r}\right|^{2}\right\}}, \gamma_{s} \geq \gamma_{t} \\
\Xi\left\{\left|\frac{\tilde{h}_{s r}}{\rho_{s r}} \sqrt{\tau P_{s}} x_{s}\right|^{2}\right\} & , \gamma_{s}<\gamma_{t} \\
\Xi\left\{\left|-\frac{\sqrt{\left(1-\rho_{s r}^{2}\right) \tau P_{s}}}{\rho_{s r}} \varepsilon_{s r} x_{s}+\left(\frac{\tilde{h}_{s r}}{\rho_{s r}}-\frac{\sqrt{1-\rho_{s r}^{2}}}{\rho_{s r}} \varepsilon_{s r}\right) \chi \sqrt{(1-\tau) P_{s}} x_{a}+n_{r}\right|^{2}\right\} &
\end{array}\right. \\
& =\left\{\begin{array}{cl}
\frac{\theta(1-\kappa) P_{s}\left|\tilde{h}_{s r}\right|^{2}}{\frac{\tau P_{s}\left|\tilde{h}_{s r}\right|^{2}}{\left(\theta \kappa+\chi^{2}[1-\theta]\right) P_{s}\left|\tilde{h}_{s r}\right|^{2}+\left(1-\rho^{2}\right) P_{s}\left(\theta+\chi^{2}[1-\theta]\right) \mu_{s r}+\rho_{s r}^{2} \sigma_{r}^{2}}} & , \gamma_{s} \geq \gamma_{t} \\
\frac{\chi^{2}(1-\tau) P_{s}\left|\tilde{h}_{s r}\right|^{2}+\left(1-\rho_{s r}^{2}\right) P_{s}\left(\tau+\chi^{2}[1-\tau]\right) \mu_{s r}+\rho_{s r}^{2} \sigma_{r}^{2}}{\chi^{2}} & , \gamma_{s}<\gamma_{t}
\end{array}\right.
\end{aligned}
$$

The knowledge of the artificial noise $x_{a}$ is merely shared among $S T, P R$, and $S R$ for securing $x_{s}$ and $x_{p}$ but $E$ is blind with it. As such, the SINRs at $E$ for recovering $x_{s}$ and $x_{p}$ are inferred from (12). Inserting (6) into (12) results in

$$
y_{e}=\left\{\begin{array}{cl}
\left(\frac{\tilde{h}_{s e}}{\rho_{s e}}-\frac{\sqrt{1-\rho_{s e}^{2}}}{\rho_{s e}} \varepsilon_{s e}\right)\left(\sqrt{\theta \kappa P_{s}} x_{p}+\sqrt{\theta(1-\kappa) P_{s}} x_{s}+\sqrt{(1-\theta) P_{s}} x_{a}\right)+n_{e} & , \gamma_{s} \geq \gamma_{t} \\
\left(\frac{\tilde{h}_{s e}}{\rho_{s e}}-\frac{\sqrt{1-\rho_{s e}^{2}}}{\rho_{s e}} \varepsilon_{s e}\right)\left(\sqrt{\tau P_{s}} x_{s}+\sqrt{(1-\tau) P_{s}} x_{a}\right)+n_{e} & , \gamma_{s}<\gamma_{t}
\end{array}\right.
$$

from which the SINRs at $E$ for restoring $x_{s}$ and $x_{p}$ are respectively derived as

$$
\begin{aligned}
& \gamma_{E s}=\left\{\begin{array}{cc}
\Xi\left\{\left|\sqrt{\theta(1-\kappa) P_{s}} x_{s} \frac{\tilde{h}_{s e}}{\rho_{s e}}\right|^{2}\right\} & , \gamma_{s} \geq \gamma_{t} \\
\Xi\left\{\left|-\frac{\sqrt{\left(1-\rho_{s e}^{2}\right) \theta(1-\kappa) P_{s}}}{\rho_{s e}} x_{s} \varepsilon_{s e}+\left(\frac{\tilde{h}_{s e}}{\rho_{s e}}-\frac{\sqrt{1-\rho_{s e}^{2}}}{\rho_{s e}} \varepsilon_{s e}\right)\left(\sqrt{\theta \kappa P_{s}} x_{p}+\sqrt{(1-\theta) P_{s}} x_{a}\right)+n_{e}\right|^{2}\right\} & , \gamma_{s}<\gamma_{t} \\
\frac{\left.\Xi\left|\frac{\tilde{h}_{s e}}{\rho_{s e}} \sqrt{\tau P_{s}} x_{s}\right|^{2}\right\}}{\Xi\left\{\left|-\frac{\sqrt{\left(1-\rho_{s e}^{2}\right) \tau P_{s}}}{\rho_{s e}} x_{s} \varepsilon_{s e}+\left(\frac{\tilde{h}_{s e}}{\rho_{s e}}-\frac{\sqrt{1-\rho_{s e}^{2}}}{\rho_{s e}} \varepsilon_{s e}\right) \sqrt{(1-\tau) P_{s}} x_{a}+n_{e}\right|^{2}\right\}} &
\end{array}\right. \\
& =\left\{\begin{array}{cl}
\frac{\theta(1-\kappa) P_{s}\left|\tilde{h}_{s e}\right|^{2}}{(\theta \kappa+1-\theta) P_{s}\left|\tilde{h}_{s e}\right|^{2}+P_{s}\left(1-\rho_{s e}^{2}\right) \mu_{s e}+\rho_{s e}^{2} \sigma_{e}^{2}} & , \gamma_{s} \geq \gamma_{t} \\
\frac{\tau P_{s}\left|\tilde{h}_{s e}\right|^{2}}{(1-\tau) P_{s}\left|\tilde{h}_{s e}\right|^{2}+P_{s}\left(1-\rho_{s e}^{2}\right) \mu_{s e}+\rho_{s e}^{2} \sigma_{e}^{2}} & , \gamma_{s}<\gamma_{t}
\end{array}\right.
\end{aligned}
$$




$$
\begin{aligned}
& \gamma_{E p}=\left\{\begin{array}{cc}
\Xi\left\{\left|\frac{\tilde{h}_{s e}}{\rho_{s e}} \sqrt{\theta \kappa P_{s}} x_{p}\right|^{2}\right\} & , \gamma_{s} \geq \gamma_{t} \\
\Xi\left\{\left|-\frac{\sqrt{\left(1-\rho_{s e}^{2}\right) \theta \kappa P_{s}}}{\rho_{s e}} x_{p} \varepsilon_{s e}+\left(\frac{\tilde{h}_{s e}}{\rho_{s e}}-\frac{\sqrt{1-\rho_{s e}^{2}}}{\rho_{s e}} \varepsilon_{s e}\right)\left(\sqrt{\theta(1-\kappa) P_{s}} x_{s}+\sqrt{(1-\theta) P_{s}} x_{a}\right)+n_{e}\right|^{2}\right\} & \\
0 & , \gamma_{s}<\gamma_{t}
\end{array}\right. \\
& =\left\{\begin{array}{cl}
\frac{\theta \kappa P_{s}\left|\tilde{h}_{s e}\right|^{2}}{(1-\theta \kappa) P_{s}\left|\tilde{h}_{s e}\right|^{2}+P_{s}\left(1-\rho_{s e}^{2}\right) \mu_{s e}+\rho_{s e}^{2} \sigma_{e}^{2}} & , \gamma_{s} \geq \gamma_{t} \\
0 & , \gamma_{s}<\gamma_{t}
\end{array}\right.
\end{aligned}
$$

It is worth emphasizing from (20) and (21) that $S T$ purposely generates the artificial noise power to corrupt the eavesdropper. Accordingly, increasing the artificial noise would secure information transmission for $x_{s}$ and $x_{p}$. Moreover, channel estimation imperfection, which is represented by terms in the denominators of (16), (18), (20), (21) weighted by $1-\rho_{\mathrm{uv}}^{2}$, degrades the performance of all receivers $(P R, S R, E)$.

The channel capacities at $P R$ and $S R$ in Stage II are inferred from (16) and (18), correspondingly, as

$$
\begin{aligned}
& C_{p}=(1-\alpha) \log \left(1+\gamma_{p}\right), \\
& C_{r}=(1-\alpha) \log \left(1+\gamma_{r}\right),
\end{aligned}
$$

where $(1-\alpha)$ is the pre-logarithm factor due to Stage II of $(1-\alpha) T$.

Similarly, the channel capacities at $E$ for decoding $x_{s}$ and $x_{p}$ in Stage II are inferred from (20) and (21), correspondingly, as

$$
\begin{aligned}
& C_{E s}=(1-\alpha) \log \left(1+\gamma_{E s}\right), \\
& C_{E p}=(1-\alpha) \log \left(1+\gamma_{E p}\right) .
\end{aligned}
$$

The secrecy capacity for $x_{s}$ is the difference between the capacities at $S R$ and $E$ for recovering $x_{s}$, i.e.,

$$
\tilde{C}_{s}=\left[C_{r}-C_{E s}\right]^{+}=(1-\alpha)\left[\log \frac{1+\gamma_{r}}{1+\gamma_{E s}}\right]^{+},
$$

where $[x]^{+}$denotes $\max (x, 0)$.

Similarly, the secrecy capacity for $x_{p}$ is the difference between the capacities at $P R$ and $E$ for restoring $x_{p}$, i.e.,

$$
\tilde{C}_{p}=(1-\alpha)\left[\log \frac{1+\gamma_{p}}{1+\gamma_{E p}}\right]^{+}
$$




\section{SOP ANALYSIS}

The SOP indicates the possibility which the secrecy capacity is below the preset security threshold $C_{0}$. Therefore, it quantifies the secrecy capability of ANaEHON. This section proposes precise closed-form SOP formulas for quickly assessing the secrecy capability for $x_{s}$ and $x_{p}$ without time-consuming simulations. Moreover, these formulas serve as a good starting point to achieve the formulas for other pivotal security measures such as IP, PSCP, STP.

\section{A. SOP for primary information $x_{p}$}

The SOP for primary information $x_{p}$ is given by

$$
S O P_{p}\left(C_{0}\right)=\operatorname{Pr}\left\{\tilde{C}_{p}<C_{0}\right\} .
$$

$S O P_{p}\left(C_{0}\right)$ is divided into two cases, dependent on whether $S T$ successfully decodes the PT's information or not:

$S O P_{p}\left(C_{0}\right)=\operatorname{Pr}\left\{\tilde{C}_{p}<C_{0} \mid C_{s} \geq C_{t}\right\} \operatorname{Pr}\left\{C_{s} \geq C_{t}\right\}+\operatorname{Pr}\left\{\tilde{C}_{p}<C_{0} \mid C_{s}<C_{t}\right\} \operatorname{Pr}\left\{C_{s}<C_{t}\right\}$.

Substituting $\tilde{C}_{p}$ in (27) into (29), one has

$$
\begin{aligned}
\operatorname{SOP}_{p}\left(C_{0}\right) & =\underbrace{\operatorname{Pr}\left\{1+\gamma_{p}<2^{C_{0} /(1-\alpha)}\left(1+\gamma_{E p}\right) \mid \gamma_{s} \geq \gamma_{t}\right\}}_{\Upsilon} \underbrace{\operatorname{Pr}\left\{\gamma_{s} \geq \gamma_{t}\right\}}_{\Delta} \\
& +\underbrace{\operatorname{Pr}\left\{\tilde{C}_{p}<C_{0} \mid \gamma_{s}<\gamma_{t}\right\}}_{\psi} \underbrace{\operatorname{Pr}\left\{\gamma_{s}<\gamma_{t}\right\}}_{1-\Delta} .
\end{aligned}
$$

The term $\Delta$ in (30) is equivalently rewritten as

$$
\Delta=\operatorname{Pr}\left\{\gamma_{s}=D\left|\tilde{h}_{p s}\right|^{2} \geq \gamma_{t}\right\}=e^{-\gamma_{t} /\left(\mu_{p s} D\right)} .
$$

In ANaEHONs, if $S T$ unsuccessfully decodes the $P T$ 's information, it does not relay the $P T$ 's information and the SINR at $P R$ for decoding $x_{p}$ is zero (i.e., $\gamma_{p}=0$ for $\gamma_{s}<\gamma_{t}$ as

shown in (16)). Therefore, in this scenario, the secrecy capacity for $x_{p}$ is also zero (i.e., $\tilde{C}_{p}=0$ conditioned on $\gamma_{s}<\gamma_{t}$ ), resulting in $\psi=1$.

The term $\Upsilon$ in (30) is rewritten after using (16) and (21) for the case of $\gamma_{s} \geq \gamma_{t}$ as

$$
\Upsilon=\operatorname{Pr}\left\{X<2^{C_{0} /(1-\alpha)} Y\right\}
$$

where

$$
X=1+\frac{A\left|\tilde{h}_{s p}\right|^{2}}{B\left|\tilde{h}_{s p}\right|^{2}+\tilde{\sigma}_{p}^{2}},
$$




$$
Y=1+\frac{A\left|\tilde{h}_{s e}\right|^{2}}{C\left|\tilde{h}_{s e}\right|^{2}+\tilde{\sigma}_{e}^{2}},
$$

with

$$
\begin{aligned}
A & =\theta \kappa P_{s}, \\
B & =\left[\theta(1-\kappa)+\chi^{2}(1-\theta)\right] P_{s}, \\
C & =(1-\theta \kappa) P_{s}, \\
\tilde{\sigma}_{p}^{2} & =\left[\theta+\chi^{2}(1-\theta)\right]\left(1-\rho_{s p}^{2}\right) \mu_{s p} P_{s}+\rho_{s p}^{2} \sigma_{p}^{2}, \\
\tilde{\sigma}_{e}^{2} & =P_{s}\left(1-\rho_{s e}^{2}\right) \mu_{s e}+\rho_{s e}^{2} \sigma_{e}^{2} .
\end{aligned}
$$

It is shown in Appendix A that $\Upsilon$ has a precise form as

$$
\Upsilon=\left\{\begin{array}{cc}
1-U e^{\frac{\tilde{\sigma}_{p}^{2}}{\mu_{s p B}}+\frac{\tilde{\sigma}_{e}^{2}}{\mu_{s e} C}} \Lambda, & N<L \\
1-U e^{\frac{\tilde{\sigma}_{p}^{2}}{\mu_{s p} B}+\frac{\tilde{\sigma}_{e}^{2}}{\mu_{s e} C}} \varphi, & 1 \leq L<N \\
1, & L<1
\end{array}\right.
$$

where

$$
\begin{aligned}
& M=1+A / B . \\
& N=1+A / C \text {. } \\
& L=2^{-C_{0} /(1-\alpha)} M, \\
& J=\frac{\tilde{\sigma}_{p}^{2} A}{\mu_{s p} B^{2}} 2^{-C_{0} /(1-\alpha)}, \\
& U=\frac{\tilde{\sigma}_{e}^{2} A}{\mu_{s e} C^{2}}, \\
& \Lambda=e^{\frac{J}{N-L}}\left\{\frac{e^{-U /(N-1)}}{U}-\frac{J e^{U /(L-N)}}{(N-L)^{2}} E i(-U V)+\right. \\
& \left.\sum_{n=2}^{\infty} \frac{J^{n}(-U)^{n-1}}{(N-L)^{2 n} n !(n-1) !}\left[e^{-\frac{U}{N-1}} \sum_{k=1}^{n-1} \frac{(k-1) !}{(-U V)^{k}}-e^{\frac{U}{L-N}} E i(-U V)\right]\right\} \\
& V=\frac{1}{N-1}-\frac{1}{N-L}, \\
& \varphi=e^{\frac{J-U}{N-L}}\left\{\frac{e^{-U V}-1}{U}+\sum_{n=1}^{\infty} \frac{J^{n}}{(N-L)^{2 n} n !}\left(\sum_{k=1}^{n-1} \frac{(-U)^{k-1} V^{k-n}}{e^{U V} \prod_{i=1}^{k}(n-i)}-\frac{(-U)^{n-1}}{(n-1) !} E i(-U V)\right),\right.
\end{aligned}
$$


with $E i(\cdot)$ being the exponential-integral function [35] built in computational softwares (e.g., Mathematica, Matlab).

Inserting $\Upsilon$ in (40), $\Delta$ in (31), and $\psi=1$ into (30) results in the precise closed-form formula of $\operatorname{SOP}_{p}\left(C_{0}\right)$.

\section{B. SOP for secondary information $x_{s}$}

The SOP for secondary information $x_{s}$ is given by

$$
S O P_{s}\left(C_{0}\right)=\operatorname{Pr}\left\{\tilde{C}_{s}<C_{0}\right\}
$$

$S O P_{s}\left(C_{0}\right)$ is divided into two cases, dependent on whether $S T$ successfully decodes the PT's information or not:

$S O P_{s}\left(C_{0}\right)=\operatorname{Pr}\left\{\tilde{C}_{s}<C_{0} \mid C_{s} \geq C_{t}\right\} \operatorname{Pr}\left\{C_{s} \geq C_{t}\right\}+\operatorname{Pr}\left\{\tilde{C}_{s}<C_{0} \mid C_{s}<C_{t}\right\} \operatorname{Pr}\left\{C_{s}<C_{t}\right\}$.

Substituting $\tilde{C}_{s}$ in (26) into (50), one has

$$
\begin{aligned}
S O P_{s}\left(C_{0}\right) & =\overbrace{\operatorname{Pr}\left\{1+\gamma_{r}<2^{C_{0} /(1-\alpha)}\left(1+\gamma_{E s}\right) \mid \gamma_{s} \geq \gamma_{t}\right\}}^{Z_{1}} \overbrace{\operatorname{Pr}\left\{\gamma_{s} \geq \gamma_{t}\right\}}^{\Delta} \\
& +\underbrace{\operatorname{Pr}\left\{1+\gamma_{r}<2^{C_{0} /(1-\alpha)}\left(1+\gamma_{E s}\right) \mid \gamma_{s}<\gamma_{t}\right\}}_{Z_{2}} \underbrace{\operatorname{Pr}\left\{\gamma_{s}<\gamma_{t}\right\}}_{1-\Delta} .
\end{aligned}
$$

The term $\Delta$ in (51) was already computed in (31) while the term $Z_{1}$ in (51) is rewritten after using (18) and (20) for the case of $\gamma_{s} \geq \gamma_{t}$ as

$$
Z_{1}=\operatorname{Pr}\left\{1+\frac{A_{1}\left|\tilde{h}_{s r}\right|^{2}}{B_{1}\left|\tilde{h}_{s r}\right|^{2}+\tilde{\sigma}_{r}^{2}}<2^{C_{0} /(1-\alpha)}\left(1+\frac{A_{1}\left|\tilde{h}_{s e}\right|^{2}}{C_{1}\left|\tilde{h}_{s e}\right|^{2}+\tilde{\sigma}_{e}^{2}}\right)\right\}
$$

where

$$
\begin{aligned}
& A_{1}=\theta(1-\kappa) P_{s}, \\
& B_{1}=\left(\theta \kappa+\chi^{2}[1-\theta]\right) P_{s}, \\
& C_{1}=(\theta \kappa+1-\theta) P_{s}, \\
& \tilde{\sigma}_{r}^{2}=\left(1-\rho_{s r}^{2}\right) P_{s}\left(\theta+\chi^{2}[1-\theta]\right) \mu_{s r}+\rho_{s r}^{2} \sigma_{r}^{2},
\end{aligned}
$$

The quantity $Z_{1}$ has the same form as $\Upsilon$ in (32). Therefore, by substituting variables appropriately into $\Upsilon$ in (32), one can achieve the precise closed-form formula of $Z_{1}$. More specifically, 
$Z_{1}$ is computed by using $\Upsilon$ in (40) with $A_{1} \rightarrow A, B_{1} \rightarrow B, C_{1} \rightarrow C, \mu_{s r} \rightarrow \mu_{s p}, \tilde{\sigma}_{r}^{2} \rightarrow \tilde{\sigma}_{p}^{2}$. As a result, the derivation of $Z_{1}$ is skipped here for compactness.

The term $Z_{2}$ in (51) is rewritten after using (18) and (20) for the case of $\gamma_{s}<\gamma_{t}$ as

$$
Z_{2}=\operatorname{Pr}\left\{1+\frac{A_{2}\left|\tilde{h}_{s r}\right|^{2}}{B_{2}\left|\tilde{h}_{s r}\right|^{2}+\breve{\sigma}_{r}^{2}}<2^{C_{0} /(1-\alpha)}\left(1+\frac{A_{2}\left|\tilde{h}_{s e}\right|^{2}}{C_{2}\left|\tilde{h}_{s e}\right|^{2}+\tilde{\sigma}_{e}^{2}}\right)\right\}
$$

where

$$
\begin{aligned}
& A_{2}=\tau P_{s}, \\
& B_{2}=\chi^{2}(1-\tau) P_{s}, \\
& C_{2}=(1-\tau) P_{s}, \\
& \breve{\sigma}_{r}^{2}=\left(1-\rho_{s r}^{2}\right) P_{s}\left(\tau+\chi^{2}[1-\tau]\right) \mu_{s r}+\rho_{s r}^{2} \sigma_{r}^{2} .
\end{aligned}
$$

The quantity $Z_{2}$ has the same form as $\Upsilon$ in (32). Therefore, by substituting variables appropriately into $\Upsilon$ in (32), one can achieve the precise closed-form formula of $Z_{2}$. More specifically, $Z_{2}$ is computed by using $\Upsilon$ in (40) with $A_{2} \rightarrow A, B_{2} \rightarrow B, C_{2} \rightarrow C, \mu_{s r} \rightarrow \mu_{s p}, \breve{\sigma}_{r}^{2} \rightarrow \tilde{\sigma}_{p}^{2}$. As a result, the derivation of $Z_{2}$ is skipped here for compactness.

Inserting the above-derived precise closed-form formulas of $\Delta, Z_{1}$, and $Z_{2}$ into (51), one achieves the precise closed-form formula of $S O P_{s}\left(C_{0}\right)$.

\section{Remarks}

The precise closed-form formulas of $S O P_{p}$ and $S O P_{s}$ are useful in quickly assessing the security measure of secondary/primary communication in ANaEHON without exhaustive simulations. Up to the best of our understanding, these formulas have not been reported yet. Moreover, they can be exploited to achieve the formulas for other pivotal security measures. More specifically, IP addresses the probability of negative secrecy capacity. Accordingly, IPs of secondary and primary communication are respectively computed as

$$
\begin{aligned}
& I P_{s}=\operatorname{Pr}\left\{\tilde{C}_{s}<0\right\}=S O P_{s}(0), \\
& I P_{p}=\operatorname{Pr}\left\{\tilde{C}_{p}<0\right\}=S O P_{p}(0) .
\end{aligned}
$$


PSCP indicates the probability of positive secrecy capacity. As such, PSCPs of secondary and primary communication are respectively expressed as

$$
\begin{aligned}
& P S C P_{s}=\operatorname{Pr}\left\{\tilde{C}_{s}>0\right\}=1-S_{S P}(0), \\
& P S C P_{p}=\operatorname{Pr}\left\{\tilde{C}_{p}>0\right\}=1-S O P_{p}(0) .
\end{aligned}
$$

Finally, STP is the product of the secrecy communication probability at a certain secrecy capacity with that secrecy capacity. Consequently, STPs of secondary and primary communication are respectively expressed as

$$
\begin{aligned}
& S T P_{s}=\left[1-\operatorname{SOP}_{s}\left(C_{0}\right)\right] C_{0} \\
& S T P_{p}=\left[1-\operatorname{SOP}_{p}\left(C_{0}\right)\right] C_{0} .
\end{aligned}
$$

\section{RESUlts AND Discussions}

The SOP of secondary/primary communication in ANaEHON is assessed through pivotal specifications. Unless otherwise stated, a set of arbitrary parameters is used to illustrate the following results: $P T$ at $(-0.6,0.2), P R$ at $(0.5,-0.2), S T$ at $(0.0,0.0), S R$ at $(0.6,0.0), E$ at $(0.6,-0.1), \eta=0.9, \sigma_{s}^{2}=\sigma_{e}^{2}=\sigma_{p}^{2}=\sigma_{r}^{2}=\tilde{\sigma}_{s}^{2}=N_{0}, \rho_{\mathrm{uv}}=\rho, \varsigma=3, P_{p} / N_{0}=10 \mathrm{~dB}$, $\alpha=\lambda=\tau=0.6, \theta=0.8, \kappa=0.7, C_{t}=C_{0}=0.1 \mathrm{bps} / \mathrm{Hz}$. Figures $2-11$ respectively denote "Sim." and "Ana." as simulated and analytical results, and demonstrate the agreement between analysis and simulation, ratifying the exactness of the analysis in (30) and (51).

Figure 2 shows the SOPs versus channel estimation imperfection reflected by $\rho$. This figure demonstrates that channel estimation error drastically affects the SOP of primary/secondary communication. More specifically, large SOPs are almost unchanged over a wide range of bad channel estimation error $(0 \leq \rho \leq 0.9)$ while $S O P_{p}$ (or $\left.S O P_{s}\right)$ significantly drops (or increases) with a slight channel estimation improvement $(0.9 \leq \rho \leq 1)$. Additionally, imperfect artificial noise cancellation at legitimate receivers degrades security performance of primary/secondary communication (i.e., SOPs increase with increasing $\chi$ ) as expected. Moreover, the SOP of primary communication is smaller than that of secondary communication at the same levels of channel estimation error and artificial noise cancellation. This is because the power allocation factor for primary and secondary signals is $\kappa=0.7$, which means that $70 \%(\kappa=0.7)$ of the ST's total transmit power allocated for legitimate information (i.e., $\theta P_{s}$ ) is for relaying the $P T$ 's information while $30 \%(1-\kappa=0.3)$ of that is for transmitting the $S T$ 's information. 


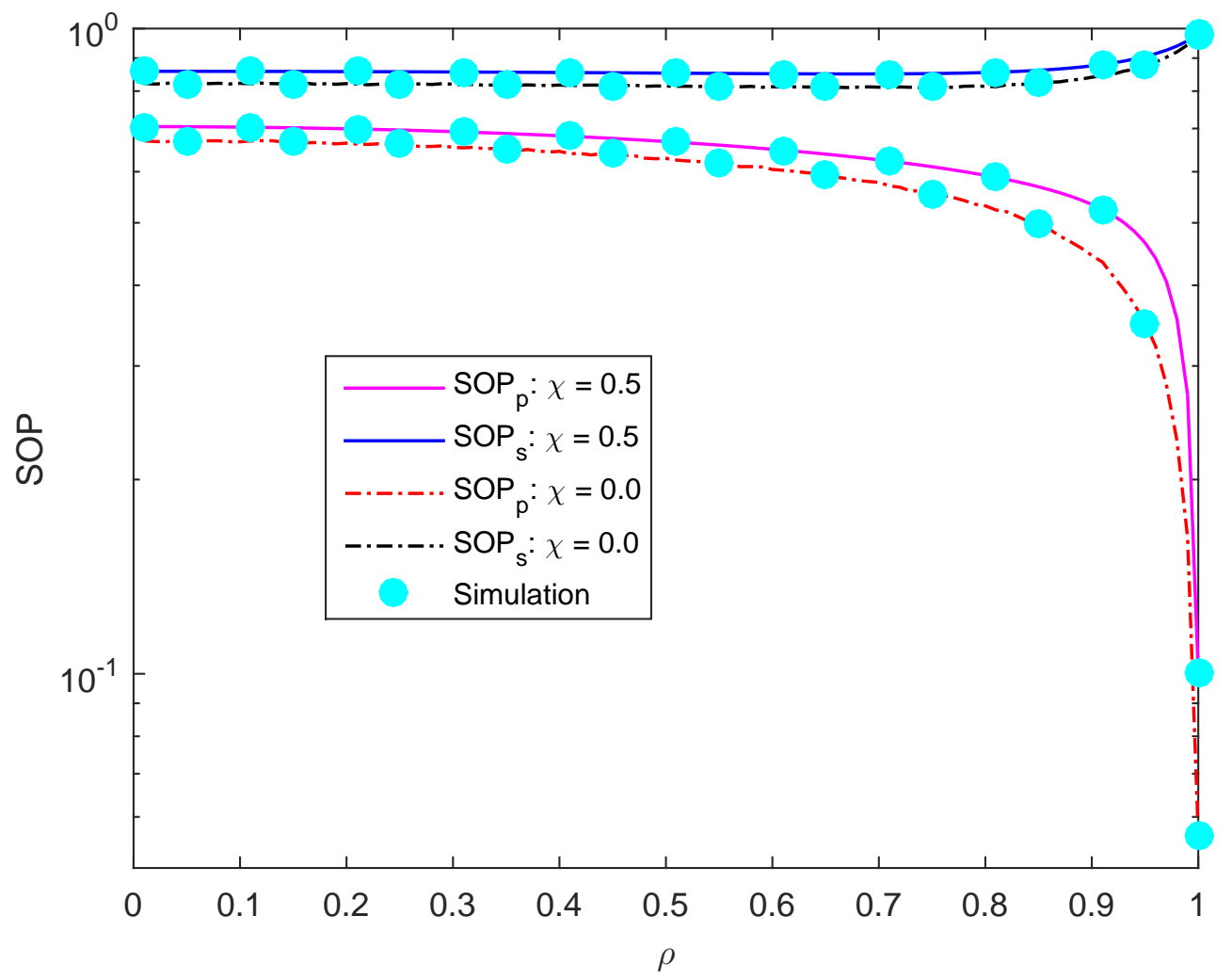

Fig. 2. SOPs versus $\rho$.

Figure 3 illustrates the SOPs versus imperfect artificial noise cancellation reflected by $\chi$. This figure shows the increase of SOPs with increasing $\chi$, which is expected because of increasing artificial noise residue at legitimate receivers. Additionally, security performance of primary communication is considerably improved (or deteriorated) with reducing channel estimation imperfection (i.e., increasing $\rho$ ) in the range of low (or high) artificial noise residue (e.g., $S O P_{p}$ at $\rho=1.0$ is smaller than $S O P_{p}$ at $\rho=0.9$ for $\chi<0.675$ but the reverse happens for $\chi>0.675$ ). Nonetheless, security performance of secondary communication is always degraded with reducing channel estimation imperfection irrespective of $\chi$ (e.g., $S O P_{s}$ at $\rho=1.0$ is larger than $S O P_{s}$ at $\rho=0.9$ for any $\chi$ ). Furthermore, due to $\kappa=0.7$ as Figure 2, primary communication is more secure than secondary communication at the same levels of channel estimation error and artificial noise cancellation, as expected.

Figure 4 shows the SOPs versus $P_{p} / N_{0}$. It is seen that owing to $\kappa=0.7$ as Figure 2, primary communication is more secure than secondary communication at the same levels of 


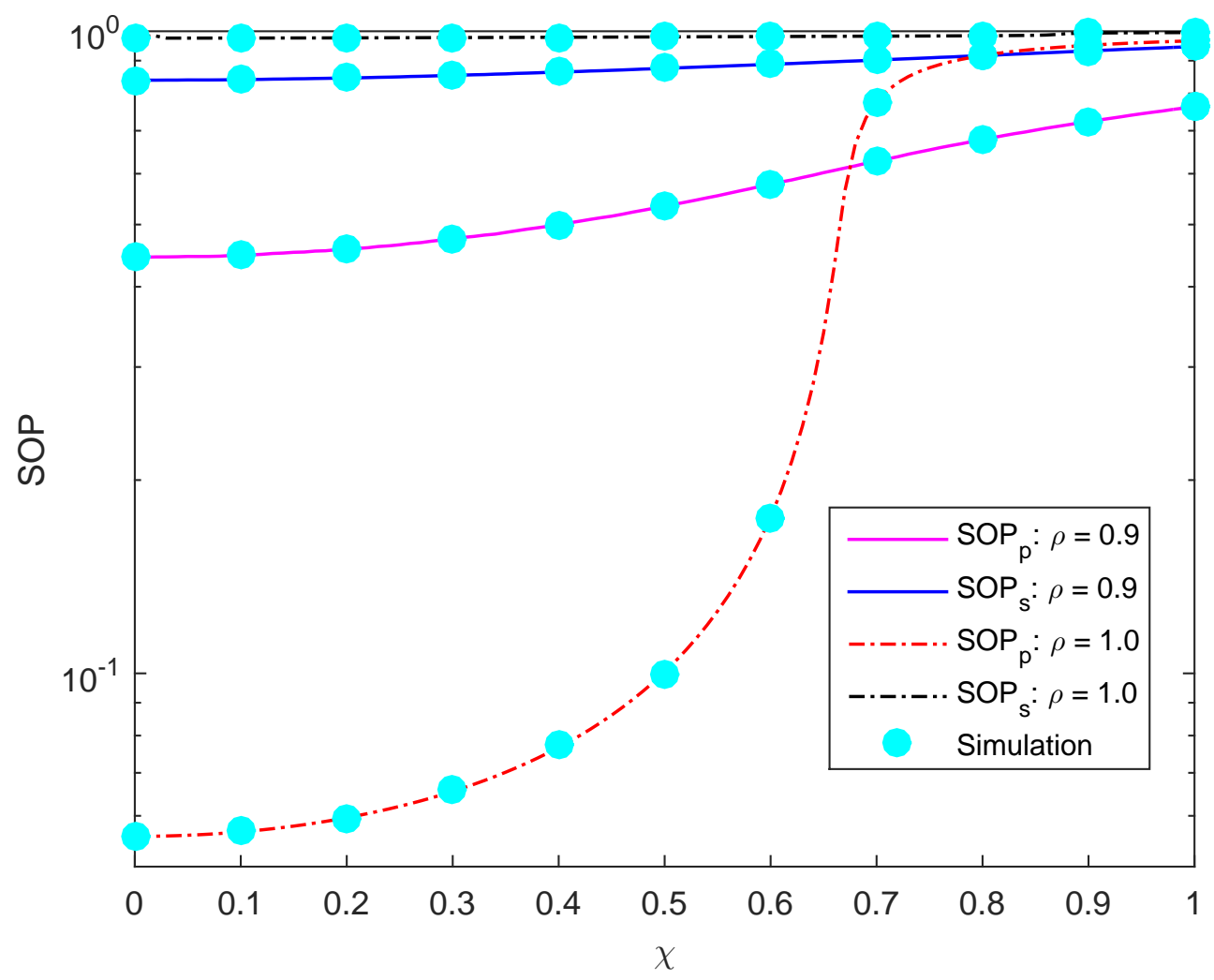

Fig. 3. SOPs versus $\chi$.

channel estimation error and artificial noise cancellation, as expected. In addition, $S O P_{p}$ is drastically reduced with better channel estimation and artificial noise cancellation, especially when the transmit power of $P T$ increases, i.e., $S O P_{p}$ at $(\rho=1.0, \chi=0.0)$ is considerably smaller than $S O P_{p}$ at $(\rho=0.9, \chi=0.5)$. Nonetheless, the reversed security performance trend is observed for secondary transmission, i.e., $S O P_{s}$ at $(\rho=1.0, \chi=0.0)$ is larger than $S O P_{s}$ at $(\rho=0.9, \chi=0.5)$. Furthermore, security performance of primary communication compromises that of secondary communication with $P_{p} / N_{0}$ (i.e., $S O P_{p}$ reduces while $S O P_{s}$ increases with $\left.P_{p} / N_{0}\right)$.

Figure 5 plots the SOPs versus $\theta$. This figure demonstrates optimum values of $\theta$, which minimize the SOP of primary/secondary communication. These optimum values balance the transmit powers for the legitimate (primary and secondary) information and the artificial noise. Moreover, $S O P_{p}$ is lower than $S O P_{s}$ at the same levels of channel estimation error and artificial noise cancellation, which can be interpreted from the fact that $\kappa=0.7$ allocates more power for 


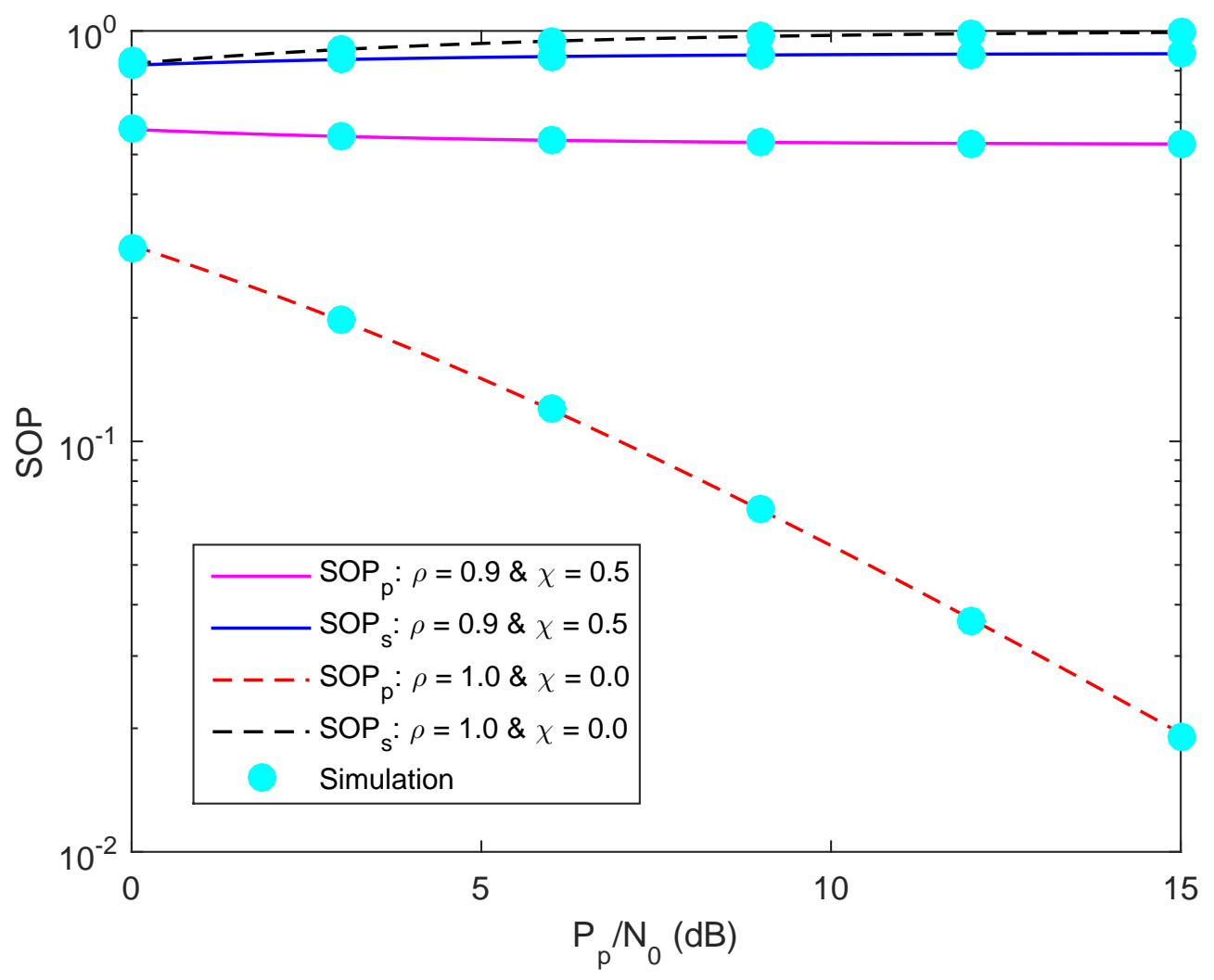

Fig. 4. SOPs versus $P_{p} / N_{0}$.

the $S T$ to transmit the $P T$ 's information than the $S T$ 's information. Furthermore, better channel estimation and artificial noise cancellation improve security performance of secondary/primary communication in a certain region of $\theta$ (e.g., $S O P_{s}$ (or $\left.S O P_{p}\right)$ at $(\rho=1.0, \chi=0.0)$ is smaller than $S O P_{s}\left(\right.$ or $\left.S O P_{p}\right)$ at $(\rho=0.9, \chi=0.5)$ when $\theta<0.575$ (or $\left.\theta<0.925\right)$ ) but degrade that performance in another region (e.g., $S O P_{s}\left(\right.$ or $\left.S O P_{p}\right)$ at $(\rho=1.0, \chi=0.0)$ is larger than $S O P_{s}$ (or $\left.S O P_{p}\right)$ at $(\rho=0.9, \chi=0.5)$ when $\theta>0.575$ (or $\left.\theta>0.925\right)$ ).

Figure 6 plots the SOPs versus $\kappa$. The results illustrate that increasing $\kappa$ improves secrecy capability of primary communication (i.e., $S O P_{p}$ decreases) while deteriorates that of secondary communication (i.e., $S O P_{s}$ increases), showing the security trade-off between secondary and primary communication. This is obvious because $\kappa$ interprets the percentage of the $S T$ 's transmit power allotted for the $P T$ 's information while $1-\kappa$ interprets the percentage of the $S T$ 's transmit power allotted for the $S T$ 's information. Therefore, increasing $\kappa$ decreases $S O P_{p}$ but increases $S O P_{s}$. Due to the conflicting security performance trend of $S O P_{p}$ and $S O P_{s}$ with respect to $\kappa$, 


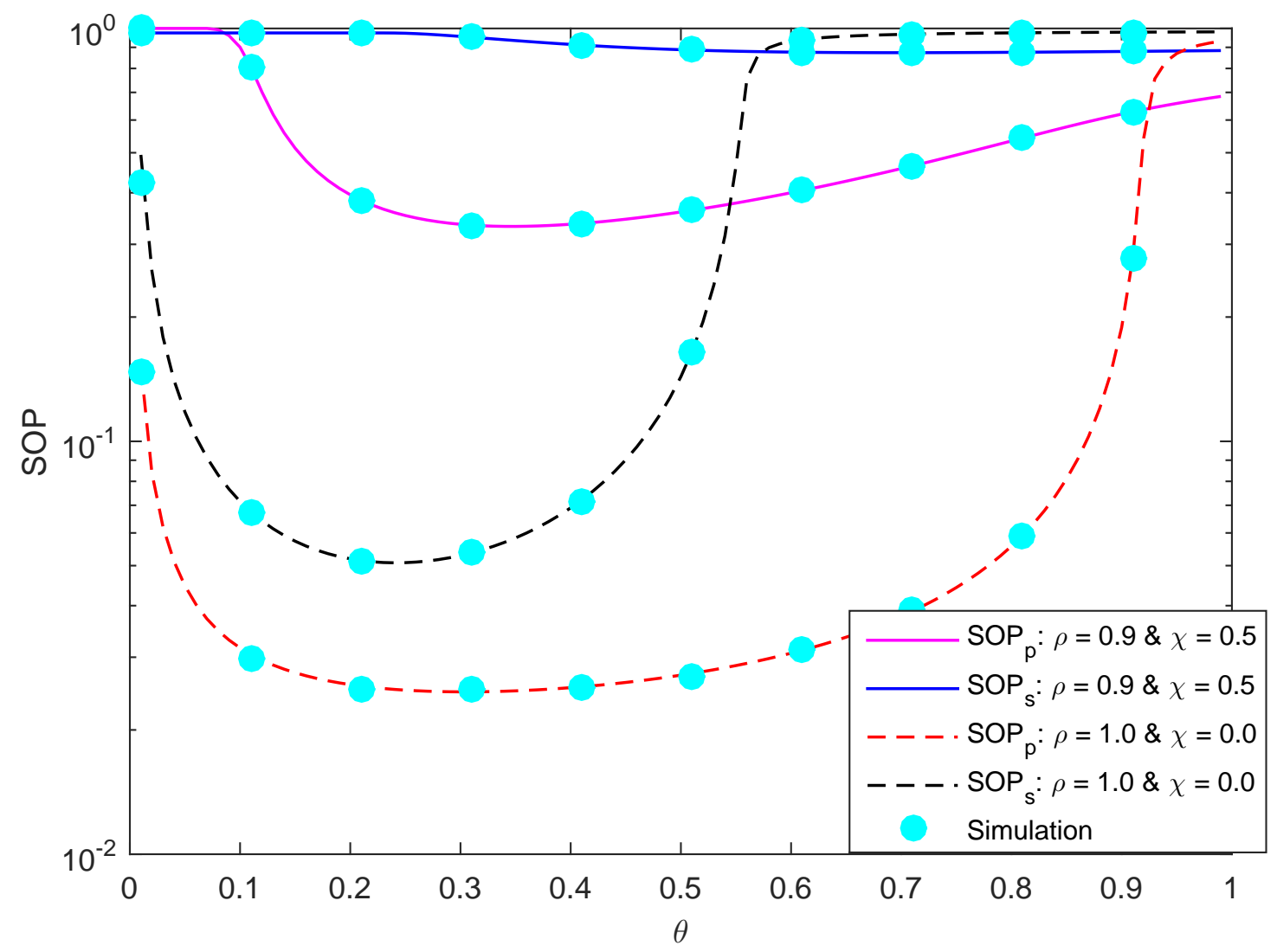

Fig. 5. SOPs versus $\theta$.

there exists a value of $\kappa$ where $S O P_{p}$ and $S O P_{s}$ are equal (e.g., $\kappa \simeq 0.535$ for $(\rho=0.9, \chi=0.5)$ and $\kappa \simeq 0.5$ for $(\rho=1.0, \chi=0.0)$ as shown in Figure 6$)$, which means the best security balance between primary and secondary communication. Moreover, the primary (or secondary) communication is in outage over a certain region of $\kappa$ (e.g., $S O P_{s}=1$ for $\kappa \geq 0.7$ and $S O P_{p}=1$ for $\kappa \leq 0.2$ when $\rho=1.0$ and $\chi=0.0$ as shown in Figure 6). Furthermore, better channel estimation and artificial noise cancellation improve security performance of primary/secondary communication in a certain region of $\kappa\left(\right.$ e.g., $S O P_{s}$ (or $\left.S O P_{p}\right)$ at $(\rho=1.0, \chi=0.0)$ is smaller than $S O P_{s}\left(\right.$ or $\left.S O P_{p}\right)$ at $(\rho=0.9, \chi=0.5)$ when $\kappa<0.52$ (or $\left.\kappa>0.48\right)$ ) but degrade that performance in another region (e.g., $S O P_{s}\left(\right.$ or $\left.S O P_{p}\right)$ at $(\rho=1.0, \chi=0.0)$ is larger than $S O P_{s}$ (or $\left.S O P_{p}\right)$ at $(\rho=0.9, \chi=0.5)$ when $0.52<\kappa<0.7$ (or $0.2<\theta<0.48$ )).

Figure 7 plots the SOPs versus $C_{t}$. It is seen that increasing $C_{t}$ improves secrecy capability of secondary communication but deteriorates that of primary communication. This is because increasing $C_{t}$ (i.e., increasing the transmission rate required by the $P T$ ) reduces the proba- 


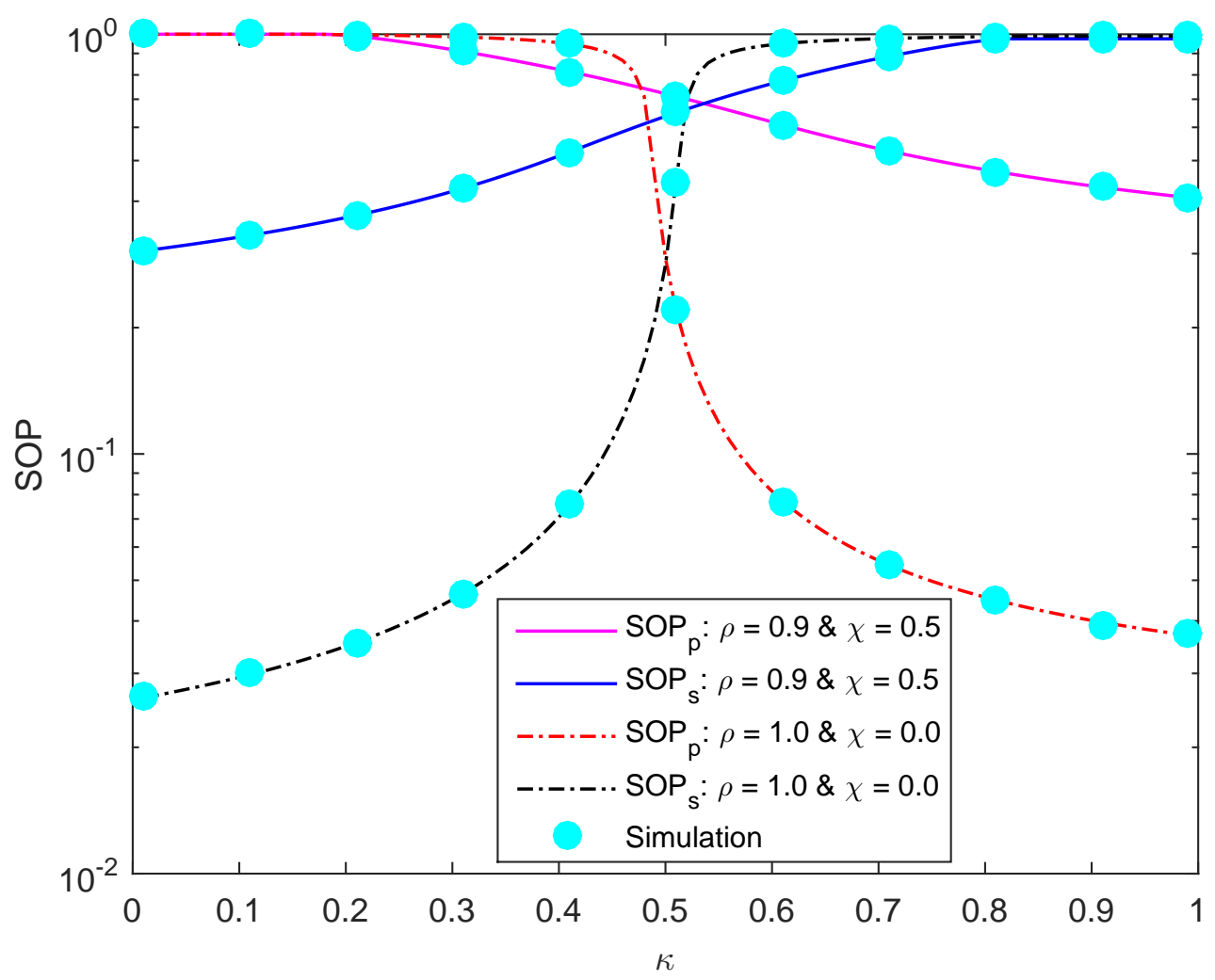

Fig. 6. SOPs versus $\kappa$.

bility of decoding successfully the PT's information at the $S T$, eventually limiting the $P T$ 's information relayed to $P R$ and increasing the $S O P_{p}$. While the $P T$ 's information is rarely relayed to $P R$ by $S T$, the information of $S T$ has more chances to be transmitted with higher transmit power, intimately reducing the $S O P_{s}$. Due to opposite security performance trends of secondary and primary networks with respect to $C_{t}$, it is possible to balance security performance for these networks by setting the appropriate required primary transmission rate; for instance, $S O P_{s}=S O P_{p}$ at $C_{t}=0.79 \mathrm{bps} / \mathrm{Hz}$ for $(\rho=0.9, \chi=0.5)$ and at $C_{t}=1.85 \mathrm{bps} / \mathrm{Hz}$ for $(\rho=1.0, \chi=0.0)$. Moreover, better channel estimation and artificial noise cancellation improve security performance of primary communication but degrades that of secondary communication, i.e., $S O P_{p}$ (or $\left.S O P_{s}\right)$ at $(\rho=1.0, \chi=0.0)$ is smaller (or larger) than $S O P_{p}$ (or $S O P_{s}$ ) at $(\rho=0.9, \chi=0.5)$.

Figure 8 plots the SOPs versus $C_{0}$. This figure exposes that increasing $C_{0}$ degrades security capability of primary/secondary communication until a complete outage, as expected. Interest- 


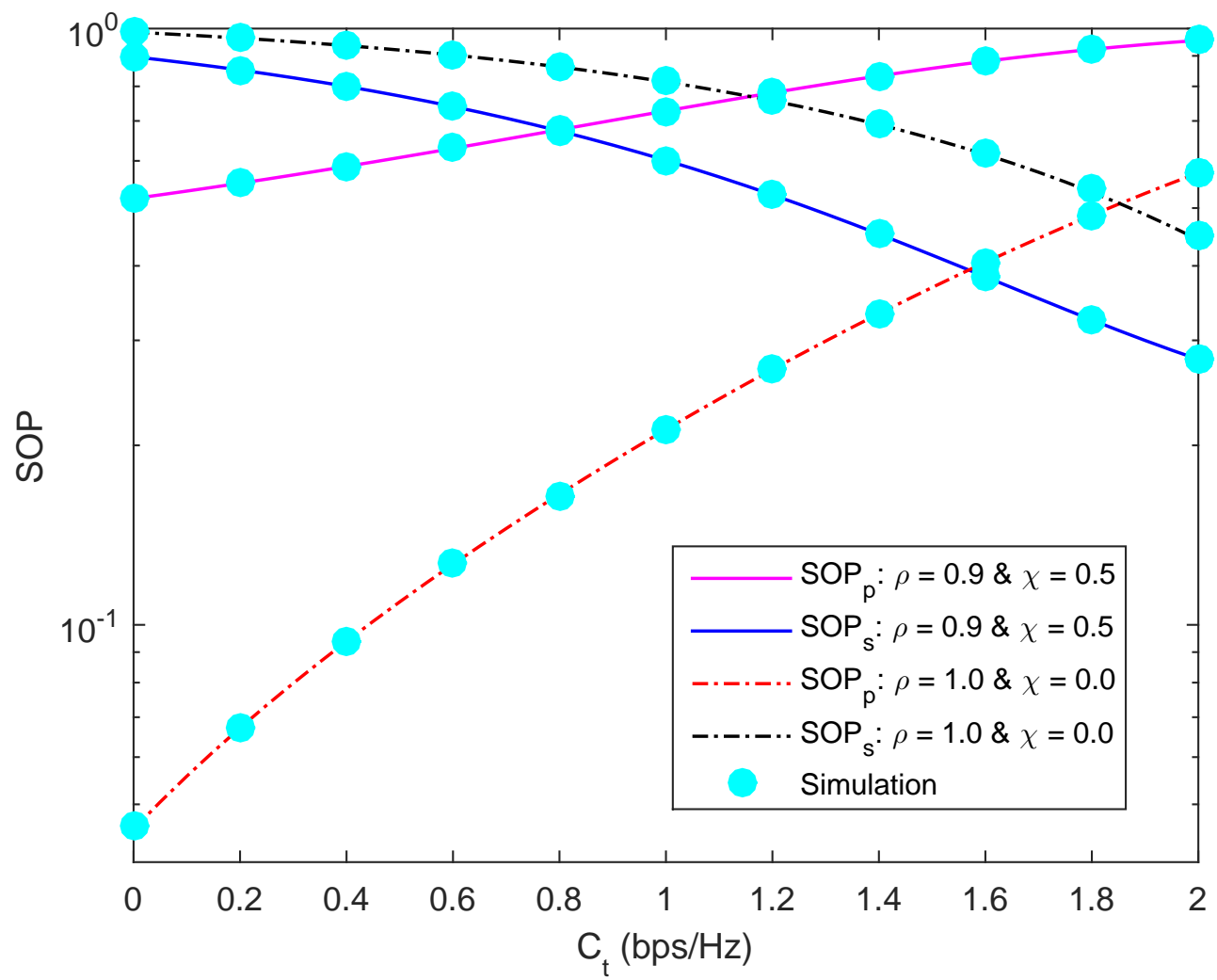

Fig. 7. SOPs versus $C_{t}$.

ingly, secrecy performance of secondary communication may be superior or inferior to that of primary communication over a certain region of $C_{0}$ (e.g., $S O P_{s}<S O P_{p}$ for $C_{0} \leq 0.016$ $\mathrm{bps} / \mathrm{Hz}$ but $S O P_{s}>S O P_{p}$ for $C_{0}>0.016 \mathrm{bps} / \mathrm{Hz}$ when $\rho=1.0$ and $\chi=0.0$ ). Moreover, better channel estimation and artificial noise cancellation also improve security performance of secondary/primary communication in a certain region of $C_{0}$; for instance, $S O P_{p}$ (or $S O P_{s}$ ) at $(\rho=1.0, \chi=0.0)$ is smaller than $S O P_{p}\left(\right.$ or $\left.S O P_{s}\right)$ at $(\rho=0.9, \chi=0.5)$ when $C_{0}$ is smaller than 0.232 (or 0.049 ) bps/Hz.

Figure 9 demonstrates the SOPs versus $\alpha$. The results expose that the security measure of primary/secondary communication can be optimized with relevant selection of $\alpha$. The optimum value of $\alpha$, which minimizes SOPs, is interpreted as follows. Increasing $\alpha$ offers the $S T$ to harvest more energy from the $P T$ and to recover successfully the $P T$ 's information with a higher probability in Stage I, thus probably enhancing security performance. Nonetheless, this increment degrades security capability in Stage II due to the decrease in secrecy capacity 


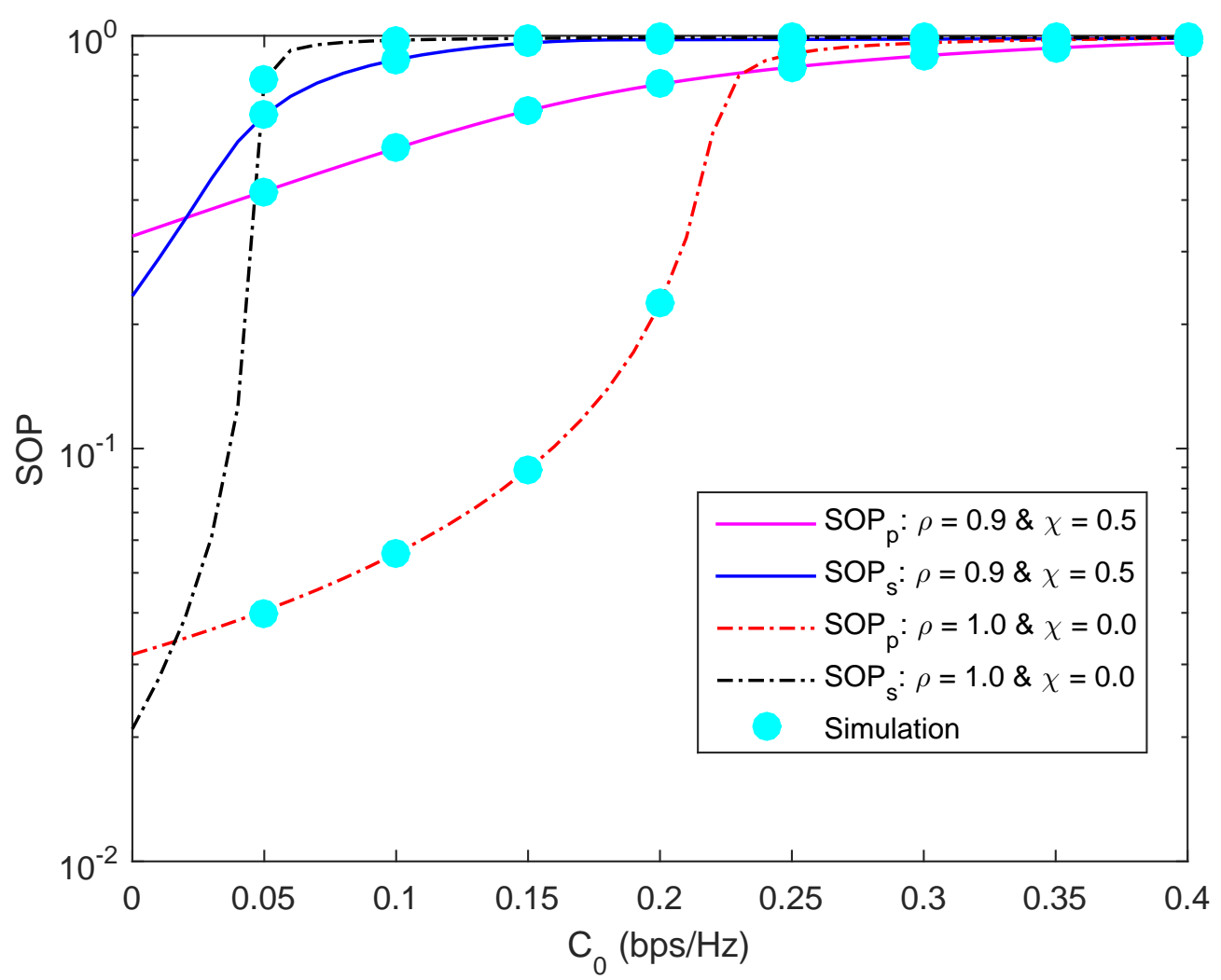

Fig. 8. SOPs versus $C_{0}$.

which is proportional to $1-\alpha$. Accordingly, $\alpha$ should be optimized to balance gains in two stages. Moreover, security performance of primary/secondary communication at the optimal value of $\alpha$ is improved with better channel estimation and artificial noise cancellation, i.e., $S O P_{p}\left(\right.$ or $\left.S O P_{s}\right)$ at the optimal value of $\alpha$ for $(\rho=1.0, \chi=0.0)$ is smaller than $S O P_{p}$ (or $\left.S O P_{s}\right)$ at the optimal value of $\alpha$ for $(\rho=0.9, \chi=0.5)$. Nonetheless, the best security of primary communication is superior to that of secondary communication at channel estimationand-artificial noise cancellation perfection (i.e., $S O P_{p}<S O P_{s}$ at the optimal value of $\alpha$ for $(\rho=1.0, \chi=0.0))$ but channel estimation-and-artificial noise cancellation imperfection reverses the performance tendency where the best security of primary communication is inferior to that of secondary communication (i.e., $S O P_{p}>S O P_{s}$ at the optimal value of $\alpha$ for $(\rho=0.9, \chi=0.5)$ ).

Figure 10 exposes the SOPs versus $\lambda$. The results show that secrecy performance of the secondary communication is almost constant and only improved for high $\lambda$ (e.g., $\lambda \geq 0.95$ ). This is because large $\lambda$ enables the $S T$ to harvest more energy from $P T$ and reduces signal 


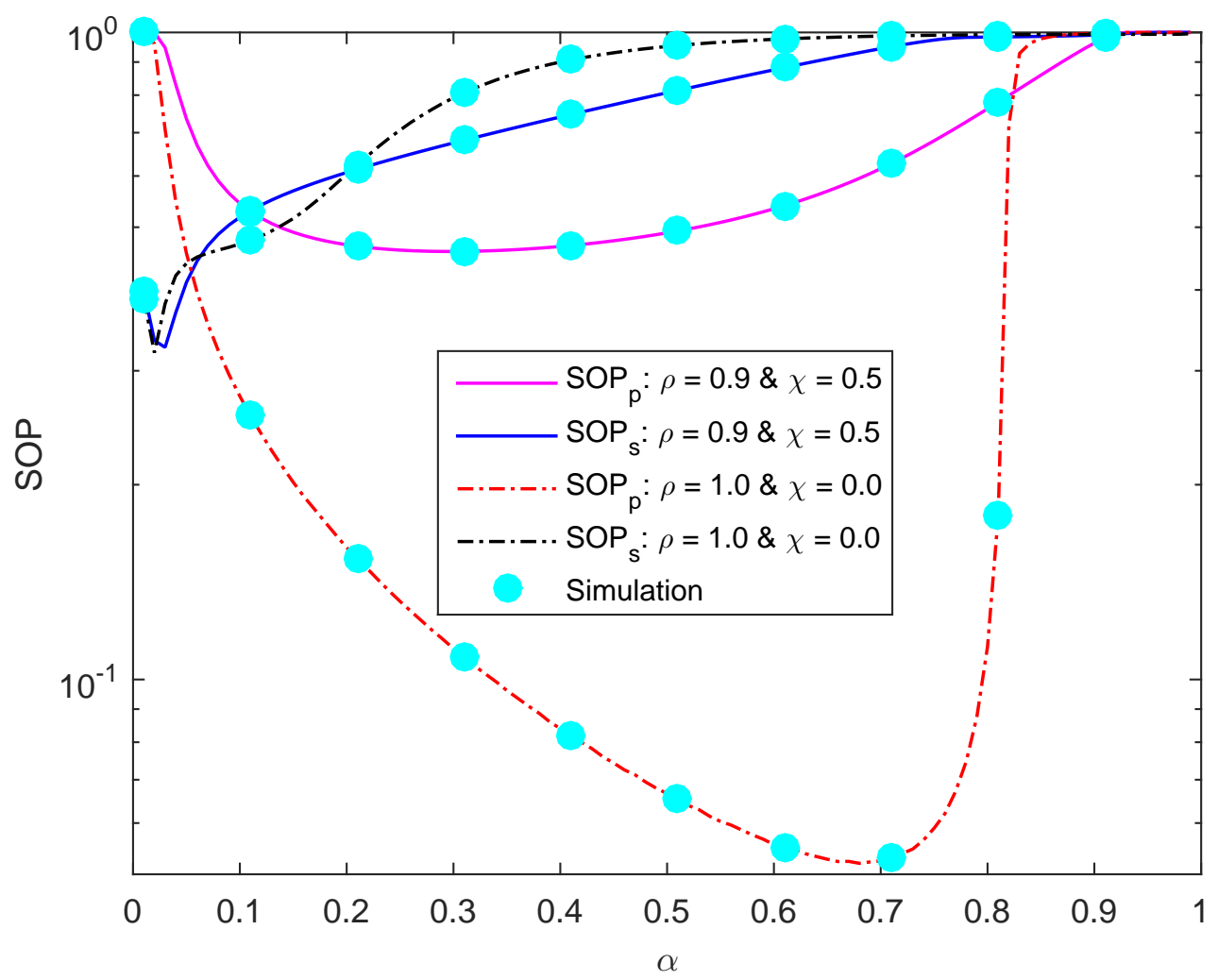

Fig. 9. SOPs versus $\alpha$.

power at the decoder of $S T$ (i.e., reduces the probability of decoding successfully the $P T$ 's information); thus, the $S T$ 's information is transmitted with higher power in Stage II, eventually declining $S O P_{s}$. Nevertheless, $\lambda$ can be selected appropriately to optimize secrecy performance of primary communication. The optimum value of $\lambda$ for minimum $S O P_{p}$ is to balance between harvested energy and the probability of decoding the PT's information at the ST. Moreover, secrecy performance of primary communication is better than that of secondary communication due to $\kappa=0.7$, which is a similar comment observed from the previous figures. Furthermore, better channel estimation and artificial noise cancellation enhance security capability of primary communication but deteriorate that of secondary communication.

It is noted that $\tau$ is the power allocation factor for artificial noise and secondary signal as the ST decodes incorrectly the PT's signal. Therefore, to observe the affect of $\tau$ clearly, it is better to consider the case that the $S T$ decodes unsuccessfully the $P T$ 's signal. This case can be set-up by selecting a large value of $C_{t}$. Figure 11 demonstrates the SOPs versus $\tau$ for 


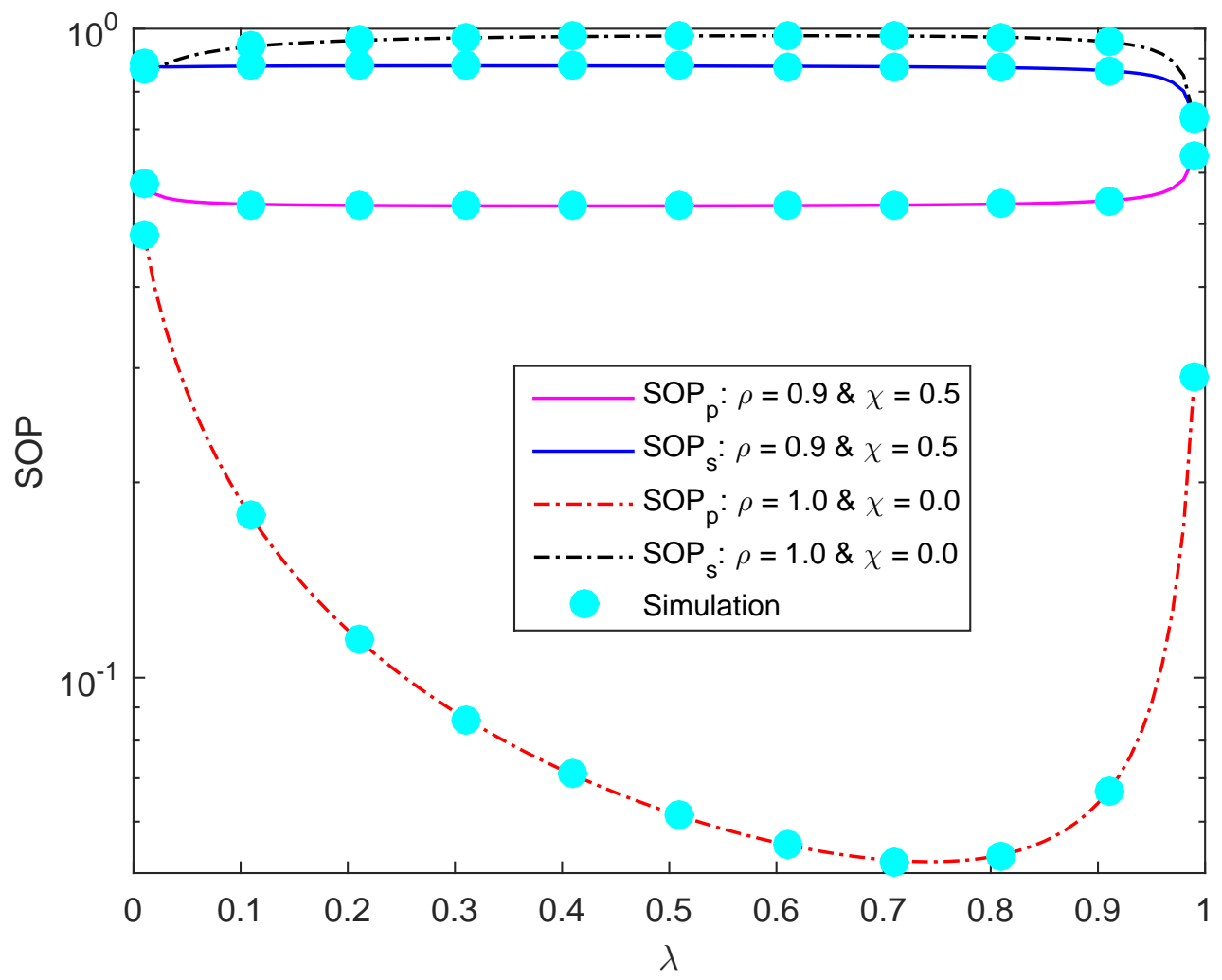

Fig. 10. SOPs versus $\lambda$.

$C_{t}=5 \mathrm{bps} / \mathrm{Hz}$. This figure exposes that primary communication is in outage because the large $C_{t}$ causes the $S T$ to fail in decoding the $P T$ 's information and hence, $P R$ does not receive it for decoding. Moreover, there exists the optimum value of $\tau$ which maximizes secrecy performance of secondary communication. This optimum $\tau$ aims to balance the power allocation for the $S T$ 's information and the artificial noise. Furthermore, better channel estimation and artificial noise cancellation enhance security performance of secondary communication, i.e., $S O P_{s}$ at $(\rho=1.0, \chi=0.0)$ is smaller than $S O P_{s}$ at $(\rho=0.9, \chi=0.5)$.

\section{CONCLUSION}

This paper implemented the overlay mechanism in cognitive radio networks where the secondary transmitter assists the information transmission of the primary transmitter as well as transmits its private information. The secondary transmitter is capable of harvesting radio frequency energy and generating the artificial noise to self-power its operation and secure primary/secondary 


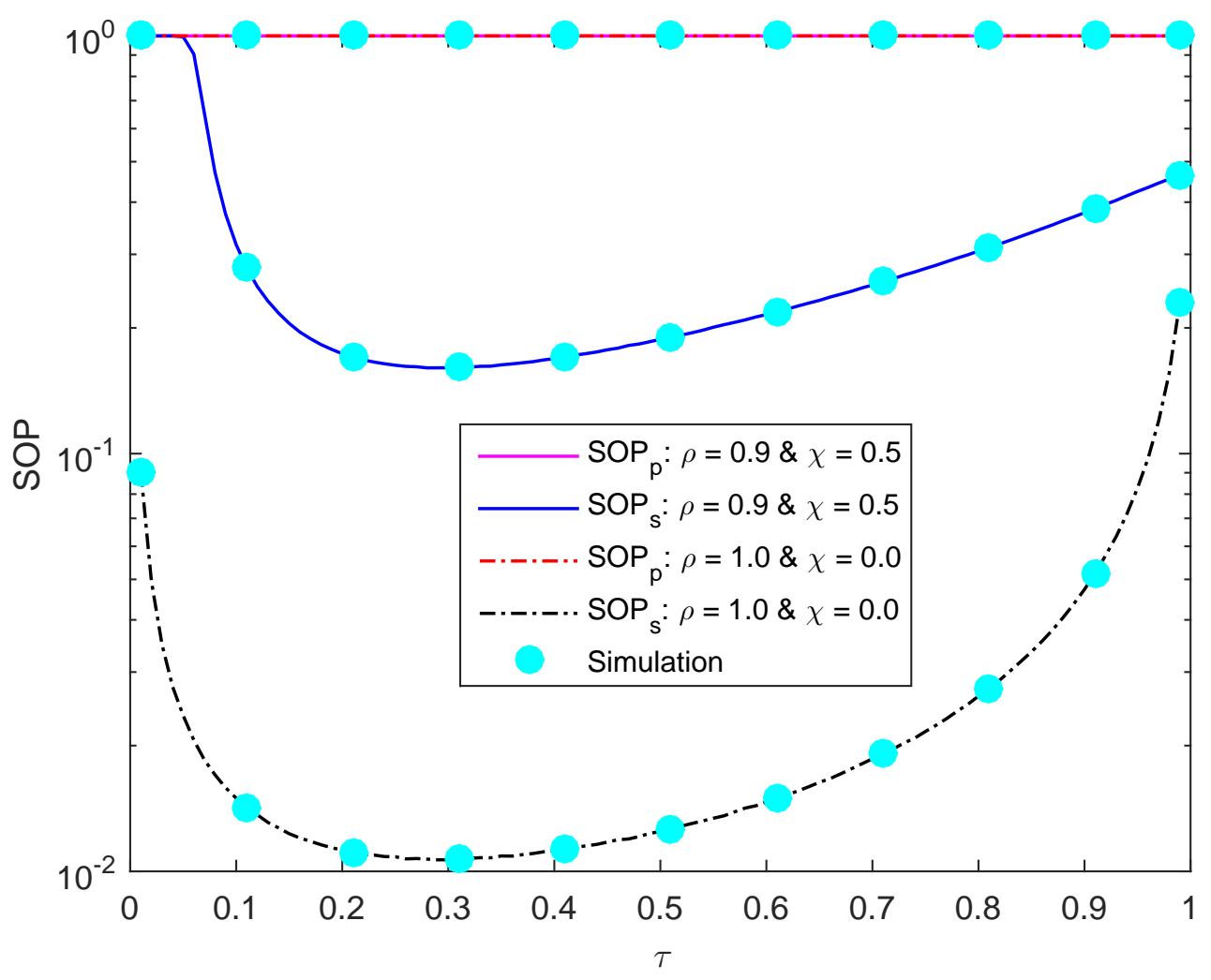

Fig. 11. SOPs versus $\tau$.

communication against eavesdroppers. Secrecy capability of primary/secondary communication is measured in terms of primary/secondary secrecy outage probability under uncertainties of channel estimation and artificial noise cancellation imperfection, which was numerically evaluated by the proposed precise closed-form formulas. Various results are generated to validate these formulas as well as shed insights into security measure of artificial noise-aided energy harvesting overlay networks with respect to main system parameters. Moreover, optimum system parameters can be found through exhaustive searches based on the proposed formulas, which well serves as a design guideline. Furthermore, the secrecy performance compromise between primary and secondary communication can be managed by adjusting system parameters appropriately. 


\section{APPENDiX A: Proof of (40)}

Before proving (40), one needs to prepare the PDFs of $X$ in (33) and $Y$ in (34). It is seen that both $X$ and $Y$ have a common form of

$$
W=1+\frac{a Z}{b Z+c},
$$

where $Z$ is the exponential random variable with the PDF of $f_{Z}(z)=\frac{1}{\mu_{Z}} e^{-\frac{z}{\mu_{Z}}}, z \geq 0$.

From (68), one infers

$$
Z=\frac{(W-1) c}{a+b-W b}
$$

Because $Z \geq 0, W$ must fall in $1 \leq W<\frac{a}{b}+1$. The Jacobian coefficient is given by

$$
\frac{d Z}{d W}=\frac{a c}{(a+b-W b)^{2}} .
$$

As such, the PDF of $W$ can be inferred from the PDF of $Z$ as

$$
f_{W}(w)=f_{Z}\left(\frac{(w-1) c}{a+b-w b}\right)\left|\frac{d Z}{d W}\right| .
$$

Inserting $f_{Z}(z)=\frac{1}{\mu_{Z}} e^{-\frac{z}{\mu_{Z}}}$ and the Jacobian coefficient into (71), one infers the PDF of $W$ as

$$
f_{W}(w)=\frac{a c}{\mu_{Z} b^{2}} \frac{e^{\frac{w-1}{w-\frac{a}{b}-1} \frac{c}{\mu_{Z}}}}{\left(w-\frac{a}{b}-1\right)^{2}}, \quad 1 \leq w \leq \frac{a}{b}+1 .
$$

Now, applying ( $a=A, b=B, c=\tilde{\sigma}_{p}^{2}, Z=\left|\tilde{h}_{s p}\right|^{2}, W=X$ ) into (72), one can obtain the PDF of $X$ as

$$
f_{X}(x)=\frac{A \tilde{\sigma}_{p}^{2}}{\mu_{s p} B^{2}} \frac{e^{\frac{x-1}{x-M} \frac{\tilde{\sigma}_{p}^{2}}{\mu_{s p} B}}}{(x-M)^{2}} \quad, 1 \leq x<M
$$

where $M$ is given in (41).

Similarly, applying ( $a=A, b=C, c=\tilde{\sigma}_{e}^{2}, Z=\left|\tilde{h}_{s e}\right|^{2}, W=Y$ ) into (72), one derives the PDF of $Y$ as

$$
f_{Y}(y)=\frac{A \tilde{\sigma}_{e}^{2}}{\mu_{s e} C^{2}} \frac{e^{\frac{y-1}{y-N} \frac{\tilde{\sigma}_{e}^{2}}{\mu_{s e C}}}}{(y-N)^{2}} \quad, 1 \leq y<N
$$

where $N$ is given in (42).

Also, before showing the proof of (40), one needs to prepare the following result:

$$
\Omega\left(a, b, B, M, \mu_{s p}, \tilde{\sigma}_{p}^{2}\right)=\int_{a}^{b} f_{X}(x) d x .
$$


Inserting $f_{X}(x)$ in (73) into (75) and after performing some variable changes, one obtains

$$
\begin{aligned}
\Omega\left(a, b, B, M, \mu_{s p}, \tilde{\sigma}_{p}^{2}\right) & =\int_{a}^{b} \frac{A \tilde{\sigma}_{p}^{2}}{\mu_{s p} B^{2}} \frac{e^{\frac{x-1}{x-M} \frac{\tilde{\sigma}_{p}^{2}}{\mu_{s p} B}}}{(x-M)^{2}} d x \\
& =\int_{a-M}^{z=x-M} \frac{A \tilde{\sigma}_{p}^{2}}{\mu_{s p} B^{2}} \frac{e^{\frac{z+M-1}{z}} \frac{\tilde{\sigma}_{p}^{2}}{\mu_{s p} B}}{z^{2}} d z \\
& =-z=\frac{A \tilde{\sigma}_{p}^{2}}{\mu_{s p} B^{2}} e^{\frac{\tilde{\sigma}_{p}^{2}}{\mu_{s p} B}} \int_{M-a}^{M-b} \frac{e^{-\frac{\tilde{\sigma}_{p}^{2}(M-1)}{\mu_{s p} B x}}}{x^{2}} d x \\
& =e^{\frac{\tilde{\sigma}_{p}^{2}}{\mu_{s p} B}}\left(e^{-\frac{\tilde{\sigma}_{p}^{2}(M-1)}{\mu_{s p} B(M-a)}}-e^{-\frac{\tilde{\sigma}_{p}^{2}(M-1)}{\mu_{s p} B(M-b)}}\right) .
\end{aligned}
$$

Now, the proof of (40) starts as follows. Because $X$ and $Y$ are independent, $\Upsilon$ in (32) is rewritten as

$$
\Upsilon=\iint_{x<2} f_{X}(x) f_{Y}(y) d x d y .
$$

Since $f_{X}(x)$ is non-zero for $1 \leq x<M$, the upper bound $2^{C_{0} /(1-\alpha)} y$ on $x$ must be considered whether it falls in $[1, M)$ or not. Therefore, $\Upsilon$ must be computed for three cases as follows:

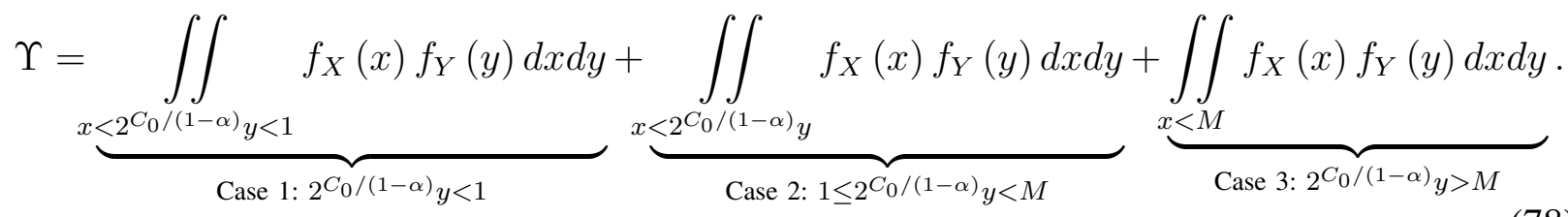

Because $f_{Y}(y)$ is non-zero for $1 \leq y<N$ and "Case 1 " is equivalent to $y<2^{-C_{0} /(1-\alpha)}<1$, $f_{Y}(y)=0$ in "Case 1" and hence, the first term in (78) is zero. Moreover, "Case 2" and "Case 3" are respectively equivalent to $2^{-C_{0} /(1-\alpha)} \leq y<L$ and $y>L$ where $L$ is given by (43). Since $f_{Y}(y)$ is non-zero for $1 \leq y<N, L$ must be considered whether it falls in $[1, N)$ or not. Therefore, three scenarios for $L$ need to be investigated as follows (please see Figure 12):

Scenario 1: $N<L$

In this scenario, the last term is (78) is rewritten as $\int_{\text {Case } 3: y>L}^{\infty}[\underbrace{\int_{=L}}\left[f_{x=1}^{M} f_{X}(x) d x\right] f_{Y}(y) d y$, which is zero because $f_{Y}(y)=0$ when $y=L>N$. Therefore, (78) is of the compact form as

$$
\Upsilon=\underbrace{\int_{y=1}^{N}\left[\int_{x=1}^{{ }^{C_{0} /(1-\alpha)} y} f_{X}(x) d x\right] f_{Y}(y) d y}_{\text {Case } 2: 2^{-C_{0} /(1-\alpha)} \leq y<L} .
$$



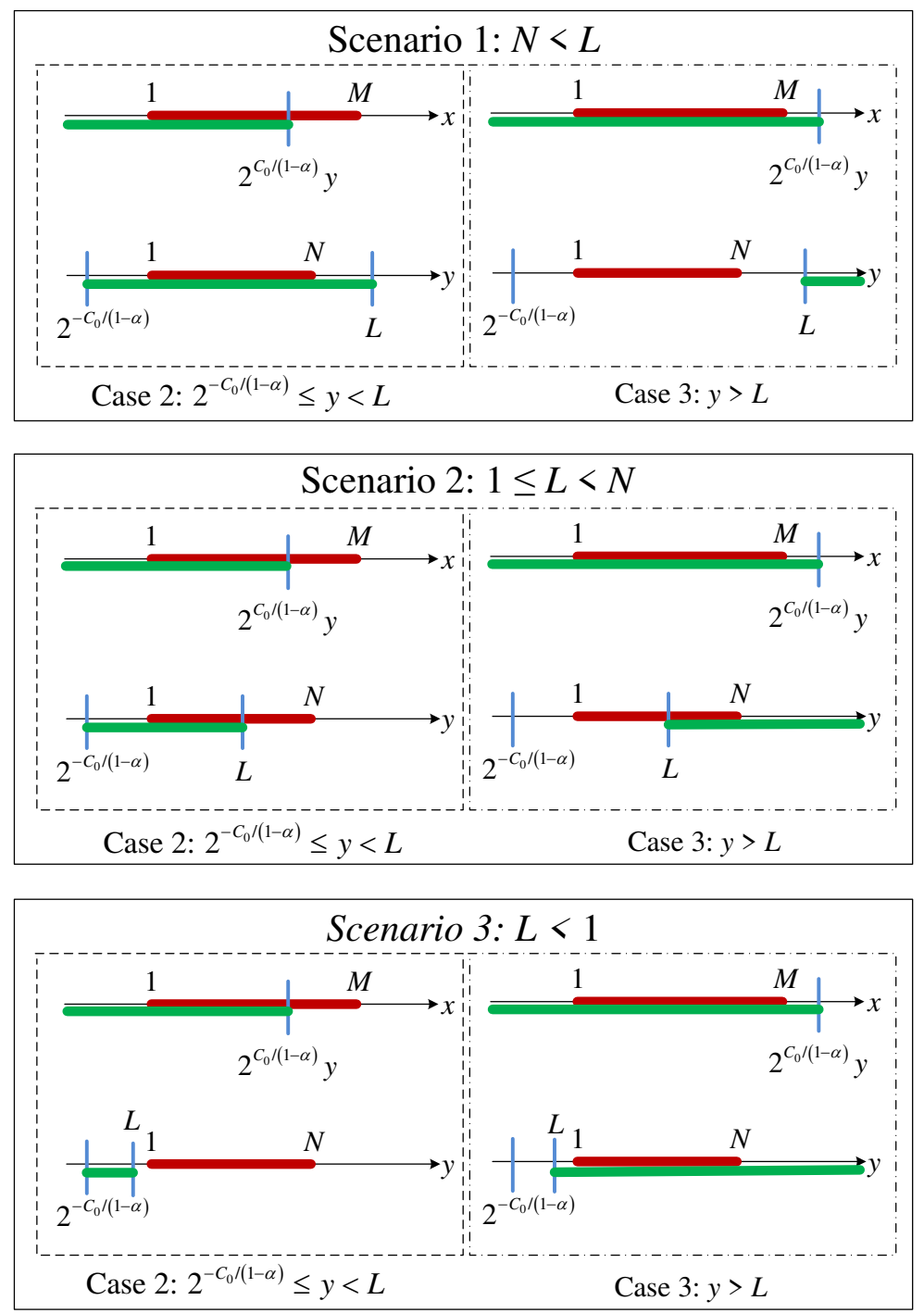

Fig. 12. Scenarios for computing $\Upsilon$.

Using (75) to compute the inner integral in (75), one obtains

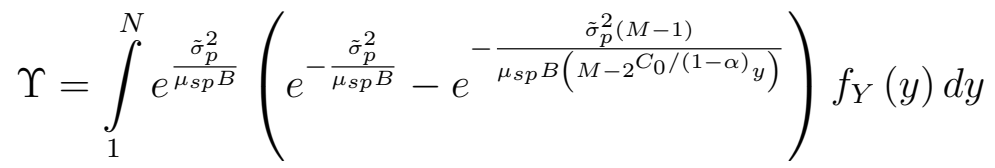

$$
\begin{aligned}
& =1-e^{\frac{\tilde{\sigma}_{p}^{2}}{\mu_{s p} B}} \int_{1}^{N} e^{\left.-\frac{\tilde{\sigma}_{p}^{2}(M-1)}{\mu_{s p} B\left(M-2 C_{0} /(1-\alpha)\right.} y\right)} f_{Y}(y) d y .
\end{aligned}
$$

It is noted that the last equality in (80) holds because $\int_{1}^{N} f_{Y}(y) d y=1$. Inserting $f_{Y}(y)$ in (74) into the last integral of (80) and after some manipulations, $\Upsilon$ in (80) matches $\Upsilon$ in (40) for 
$N<L$ where

$$
\Lambda=\int_{1}^{N} \frac{e^{\frac{J}{y-L}+\frac{U}{y-N}}}{(y-N)^{2}} d y .
$$

with $J$ and $U$ being given in (44) and (45), respectively.

Therefore, to complete the proof of $\Upsilon$ for $N<L$, one must prove that (81) matches (46). Towards this end, some appropriate variable changes are applied to reduce (81) to

$$
\begin{aligned}
\Lambda & \stackrel{x=1 /(y-N)}{=}-\int_{1 /(1-N)}^{-\infty} e^{\frac{J}{N-L+1 / x}+U x} d x \\
& \stackrel{y=-x}{=} \int_{1 /(N-1)}^{\infty} e^{\frac{J}{N-L} \frac{y}{y-1 /(N-L)}-U y} d y \\
& =e^{\frac{J}{N-L}} \int_{1 /(N-1)}^{\infty} e^{\frac{J /(N-L)^{2}}{y-1 /(N-L)}-U y} d y .
\end{aligned}
$$

Performing the series expansion $e^{x}=\sum_{n=0}^{\infty} \frac{x^{n}}{n !}$ for the term $e^{\frac{J /(N-L)^{2}}{y-1 /(N-L)}}$ in (82) results in

$$
\begin{aligned}
\Lambda & =e^{\frac{J}{N-L}} \int_{1 /(N-1)}^{\infty} e^{-U y}\left(\sum_{n=0}^{\infty} \frac{1}{n !}\left[\frac{J /(N-L)^{2}}{y-1 /(N-L)}\right]^{n}\right) d y \\
& =e^{\frac{J}{N-L}} \sum_{n=0}^{\infty} \frac{J^{n}}{(N-L)^{2 n} n !} \int_{1 /(N-1)}^{\infty} \frac{e^{-U y}}{[y-1 /(N-L)]^{n}} d y \\
& =e^{\frac{J}{N-L}}\left\{\int_{1 /(N-1)}^{\infty} e^{-U y} d y+\frac{J}{(N-L)^{2}} \int_{1 /(N-1)}^{\infty} \frac{e^{-U y}}{y+1 /(L-N)} d y\right. \\
& \left.+\sum_{n=2}^{\infty} \frac{J^{n}}{(N-L)^{2 n} n !} \int_{1 /(N-1)}^{\infty} \frac{e^{-U y}}{[y+1 /(L-N)]^{n}} d y\right\} .
\end{aligned}
$$

The first integral in the last equality of (83) is straightforwardly computed. Also, based on the definition of the exponential-integral function, the second integral in the last equality of (83) is solved easily. The last integral in (83) is evaluated in closed-form with the help of [35, eq. (3.353.1)]. After computing these three integrals, one reduces (83) to (46). This finishes the proof of $\Upsilon$ for $N<L$.

Scenario 2: $1 \leq L<N$ 
In this scenario, (78) is rewritten after noting that $1 \leq L<N$ :

$$
\Upsilon=\underbrace{\int_{y=2^{-C_{0} /(1-\alpha)}}^{L}\left[\int_{x=1}^{2^{C_{0} /(1-\alpha)} y} f_{X}(x) d x\right] f_{Y}(y) d y}_{\text {Case } 2: 2^{-C_{0} /(1-\alpha)} \leq y<L}+\underbrace{\int_{y=L}^{N}\left[\int_{x=1}^{M} f_{X}(x) d x\right] f_{Y}(y) d y}_{\text {Case 3: } y>L} .
$$

Before computing (84), it is worth noting that $f_{X}(x)$ is non-zero for $x \in[1, M)$ and hence, $\int_{x=1}^{M} f_{X}(x) d x=1$. Also, the integral $\int_{x=1}^{2^{C_{0} /(1-\alpha)} y} f_{X}(x) d x$ can be solved as $\Omega\left(1,2^{C_{0} /(1-\alpha)} y, B, M, \mu_{s p}, \tilde{\sigma}_{p}^{2}\right)$ according to (75). Therefore, the compact form of (84) is as follows:

$$
\begin{aligned}
& \Upsilon=\int_{1}^{L} \Omega\left(1,2^{C_{0} /(1-\alpha)} y, B, M, \mu_{s p}, \tilde{\sigma}_{p}^{2}\right) f_{Y}(y) d y+\int_{L}^{N} f_{Y}(y) d y \\
& =\int_{1}^{L} e^{\frac{\tilde{\sigma}_{p}^{2}}{\mu_{s p} B}}\left(e^{-\frac{\tilde{\sigma}_{p}^{2}}{\mu_{s p} B}}-e^{\left.-\frac{\tilde{\sigma}_{p}^{2}(M-1)}{\mu_{s p} B\left(M-2 C_{0} /(1-\alpha)\right.} y\right)}\right) f_{Y}(y) d y+\int_{L}^{N} f_{Y}(y) d y
\end{aligned}
$$

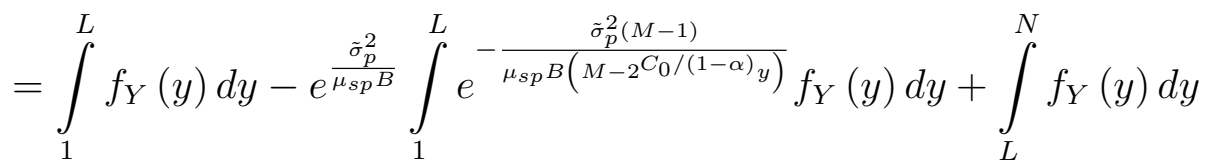

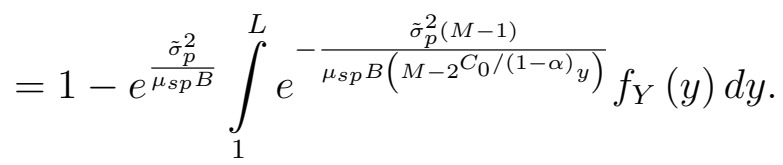

It is noted that the last equality in (85) is obtained because $\int_{1}^{N} f_{Y}(y) d y=1$. Inserting $f_{Y}(y)$ in (74) into the last integral of (85) and after some manipulations, $\Upsilon$ in (85) is exactly the same as (40) for $1 \leq L<N$ where

$$
\varphi=\int_{1}^{L} \frac{e^{J /(y-L)+U /(y-N)}}{(y-N)^{2}} d y .
$$

To complete the proof of $\Upsilon$ for $1 \leq L<N$, one must prove that (86) matches (48). Towards this end, some appropriate variable changes and the series expansion (similarly to steps in (82) and (83)) are applied to reduce (86) to

$$
\begin{aligned}
\varphi & =e^{\frac{J-U}{N-L}} \sum_{n=0}^{\infty} \frac{J^{n}}{(N-L)^{2 n} n !} \int_{V}^{0} \frac{e^{-U x}}{x^{n}} d x \\
& =e^{\frac{J-U}{N-L}}\left\{\int_{V}^{0} e^{-U x} d x+\sum_{n=1}^{\infty} \frac{J^{n}}{(N-L)^{2 n} n !} \int_{V}^{0} \frac{e^{-U x}}{x^{n}} d x\right\}
\end{aligned}
$$


The first integral in (87) is straightforwardly computed while the second one is computed with the aid of [35, eq. (2.324.2)]. Plugging the results of these two integrals into (87), one reduces (87) to (48). This finishes the proof of $\Upsilon$ for $1 \leq N<L$.

Scenario 3: $L<1$

In this scenario, (78) is rewritten after noting that $L<1$ :

$$
\Upsilon=\underbrace{\int_{y=2^{-C_{0} /(1-\alpha)}}^{L}\left[\int_{x=1}^{2^{C_{0} /(1-\alpha)} y} f_{X}(x) d x\right] f_{Y}(y) d y}_{\text {Case } 2: 2^{-C_{0} /(1-\alpha)} \leq y<L}+\underbrace{\int_{y=1}^{N}\left[\int_{x=1}^{M} f_{X}(x) d x\right] f_{Y}(y) d y}_{\text {Case 3: } y>L} .
$$

Because $f_{Y}(y)=0$ when $y=L<1$, the first term in (88) is zero. Moreover, $f_{X}(x)$ is non-zero for $1 \leq x<M$ and $f_{Y}(y)$ is non-zero for $1 \leq y<N$ and hence, the second term in (88) is one. Plugging these results into (88), one infers $\Upsilon=1$, which coincides with (40) for $L<1$, finishing the proof of $\Upsilon$ for $L<1$.

By integrating three above scenarios, one can prove that $\Upsilon$ is exactly represented as (40).

\section{REFERENCES}

[1] M. Wazid et al., "Security in 5G-Enabled Internet of Things Communication: Issues, Challenges, and Future Research Roadmap," IEEE Access, vol. 9, pp. 4466-4489, Dec. 2020.

[2] U. Gustavsson et al., "Implementation Challenges and Opportunities in Beyond-5G and 6G Communication,” IEEE Journal of Microwaves, vol. 1, no. 1, pp. 86-100, Jan. 2021.

[3] O. L. A. Lpez et al., "Massive Wireless Energy Transfer: Enabling Sustainable IoT Towards 6G Era," IEEE IoT Journal, accepted.

[4] B. Djamaa et al., "Efficient and Stateless P2P Routing Mechanisms for the Internet of Things," IEEE IoT Journal, accepted.

[5] M. Ali et al., "LTE-U WiFi HetNets: Enabling Spectrum Sharing for 5G/Beyond 5G Systems," IEEE IoT Mag., vol. 3, no. 4, pp. 60-65, Dec. 2020.

[6] K. Ho-Van et al., "Security Enhancement for Energy Harvesting Cognitive Networks with Relay Selection," Wire. Commun. and Mobile Computing, vol. 2020, Article ID 8867148, pp. 1-13.

[7] M. Koca et al., "Empirical Feasibility Analysis for Energy Harvesting Intravehicular Wireless Sensor Networks," IEEE IoT Journal, vol. 8, no. 1, pp. 179-186, Jan. 2021.

[8] T. Le Anh et al., "Secrecy Performance of a Multi-NOMA-MIMO System in the UEH Relaying Network Using the PSO Algorithm,” IEEE Access, vol. 9, pp. 2317-2331, Dec. 2020.

[9] Y. Nie et al., "Achievable Rate Region of Energy-Harvesting Based Secure Two-Way Buffer-Aided Relay Networks," IEEE Trans. Info. Forensics and Security, vol. 16, pp. 1610-1625, Nov. 2020.

[10] E. Stai et al., "Optimal Resource Allocation in Multihop Wireless Networks Relying on Energy Harvesting," IEEE Commun. Lett., vol. 25, no. 1, pp. 224-228, Jan. 2021.

[11] I. Budhiraja et al., "SWIPT-enabled D2D Communication Underlaying NOMA-Based Cellular Networks in Imperfect CSI," IEEE Trans. Veh. Tech., accepted. 
[12] B. Kim et al., "Joint Channel Estimation, Training Design, Tx Power Allocation, and Rx Power Splitting for MIMO SWIPT Systems," IEEE Commun. Lett., accepted.

[13] D. Masotti et al., "RF Systems Design for Simultaneous Wireless Information and Power Transfer (SWIPT) in Automation and Transportation,” IEEE Journal of Microwaves, vol. 1, no. 1, pp. 164-175, Jan. 2021.

[14] A. Prathima et al., "Performance Analysis and Optimization of Bidirectional Overlay Cognitive Radio Networks With Hybrid-SWIPT,” IEEE Trans. Veh. Tech., vol. 69, no. 11, pp. 13467-13481, Nov. 2020.

[15] S. Thapar et al., "Decoding Orders for Securing Untrusted NOMA," IEEE Networking Lett., accepted.

[16] Z. Abdullah et al., "Enhanced Secrecy Performance of Multihop IoT Networks With Cooperative Hybrid-Duplex Jamming," IEEE Trans. Info. Forensics and Security, vol. 16, pp. 161-172, Jun. 2020.

[17] F. Wang et. al., "Secure Resource Allocation for Polarization-Based Non-Linear Energy Harvesting Over 5G Cooperative CRNs," IEEE Wire. Commun. Lett., accepted.

[18] R. Su et al., "Destination-Assisted Jamming for Physical-Layer Security in SWIPT Cognitive Radio Systems," in Proc. IEEE WCNC, Barcelona, Spain, 2018, pp. 1-6.

[19] R. Su et al., "Secure Cooperative Transmission in Cognitive AF Relay Systems with Destination-Aided Jamming and Energy Harvesting,” in Proc. IEEE PIMRC, Istanbul, Turkey, 2019, pp. 1-5.

[20] D. Wang et al., "Primary Privacy Preserving With Joint Wireless Power and Information Transfer for Cognitive Radio Networks,” IEEE Trans. Cogn. Commun. and Networking, vol. 6, no. 2, pp. 683-693, Jun. 2020.

[21] M. Xu et al., "Secure Transmission Solutions in Energy Harvesting Enabled Cooperative Cognitive Radio Networks," in Proc. IEEE WCNC, Barcelona, Spain, 2018, pp. 1-6.

[22] D. Wang and C. Tellambura, "Performance Analysis of Energy Beamforming WPCN Links With Channel Estimation Errors,” IEEE OJ-CS, vol. 1, pp. 1153-1170, Jul. 2020.

[23] M. Li et al. "Physical Layer Security in Overlay Cognitive Radio Networks With Energy Harvesting," IEEE Trans. Veh. Tech., vol. 67, pp. 11274-11279, Sep. 2018.

[24] H. Dang-Ngoc et al., "Key Secrecy Performance Metrics of Overlay Networks with Energy Scavenging and Artificial Noise,” in Proc. IEEE SigTelCom, Hanoi, Vietnam, 28-29 Aug. 2020, pp. 77-81.

[25] N. Pham-Thi-Dan et al., "Secrecy Throughput Analysis of Energy Scavenging Overlay Networks with Artificial Noise," in Proc. IEEE ATC, Nha Trang, Vietnam, 8-10 Aug. 2020, pp. 90-94.

[26] L. Chen et al., "Primary Secrecy Is Achievable: Optimal Secrecy Rate in Overlay CRNs with an Energy Harvesting Secondary Transmitter," in Proc. ICCCN, Las Vegas, USA, 2015, pp. 1-6.

[27] F. Benkhelifa et al., "A Thresholding-based Antenna Switching in MIMO Cognitive Radio Networks with SWIPT-enabled Secondary Receiver," in Proc. IEEE ICC, Paris, France, 21-25 May 2017, pp. 1-6.

[28] X. Zhou et al., "Wireless Information and Power Transfer: Architecture Design and Rate-Energy Tradeoff," IEEE Trans. Commun., vol. 61, no. 11, pp. 4754-4767, Nov. 2013.

[29] K. Ho-Van et al., "Security Performance of Underlay Cognitive Relaying Networks with Energy Harvesting," Wire. Per. Commun., vol. 110, no. 2, pp. 829-846, Jan. 2020.

[30] K. Ho-Van et al., "Security Analysis for Underlay Cognitive Network with Energy Scavenging Capable Relay over Nakagami-m Fading Channels,” Wire. Commun. and Mobile Computing, vol. 2019, Article ID 5080952, pp. 1-16.

[31] P. Nguyen-Huu et al., "Secrecy Outage Analysis of Energy Harvesting Two-Way Relaying Networks with Friendly Jammer," IET Commun., vol. 13, no. 13, pp. 18771885, Aug. 2019.

[32] L. Ge et. al., "Performance Analysis for Multihop Cognitive Radio Networks With Energy Harvesting by Using Stochastic Geometry," IEEE IoT Journal, vol. 7, no. 2, pp. 1154-1163, Feb. 2020. 
[33] S. Solanki et. al., "Performance Analysis of Piece-Wise Linear Model of Energy Harvesting-Based Multiuser Overlay Spectrum Sharing Networks,” IEEE OJ-CS, vol. 1, pp. 1820-1836, Nov. 2020.

[34] S. Bayat et. al., "Resource Allocation for MC MISO-NOMA SWIPT-Enabled HetNets With Non-Linear Energy Harvesting," IEEE Access, vol. 8, pp. 192270-192281, Oct. 2020.

[35] I. S. Gradshteyn and I. M. Ryzhik, Table of Integrals, Series and Products, 6th ed. San Diego, CA: Academic, 2000. 
Figures

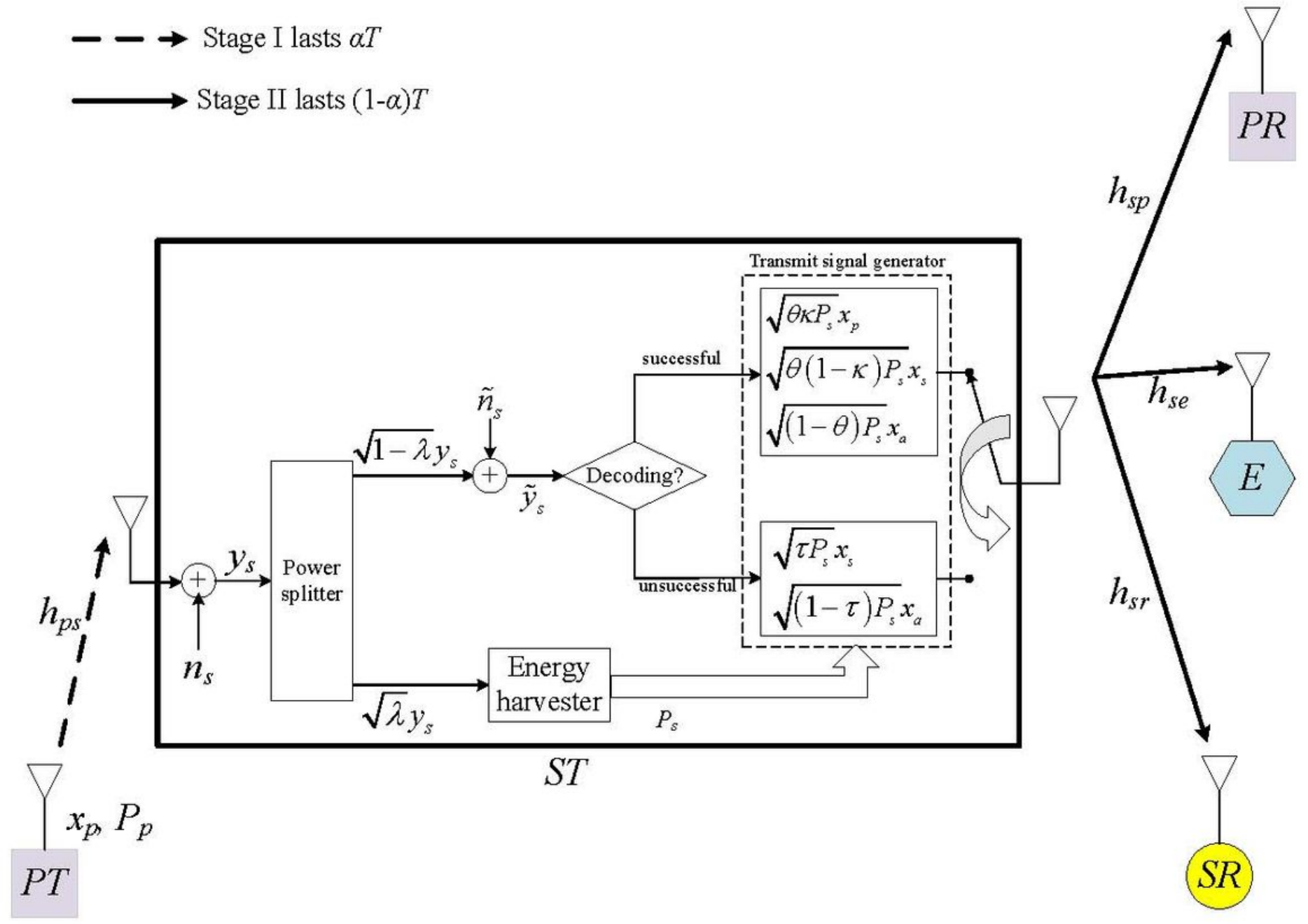

Figure 1

System model. 


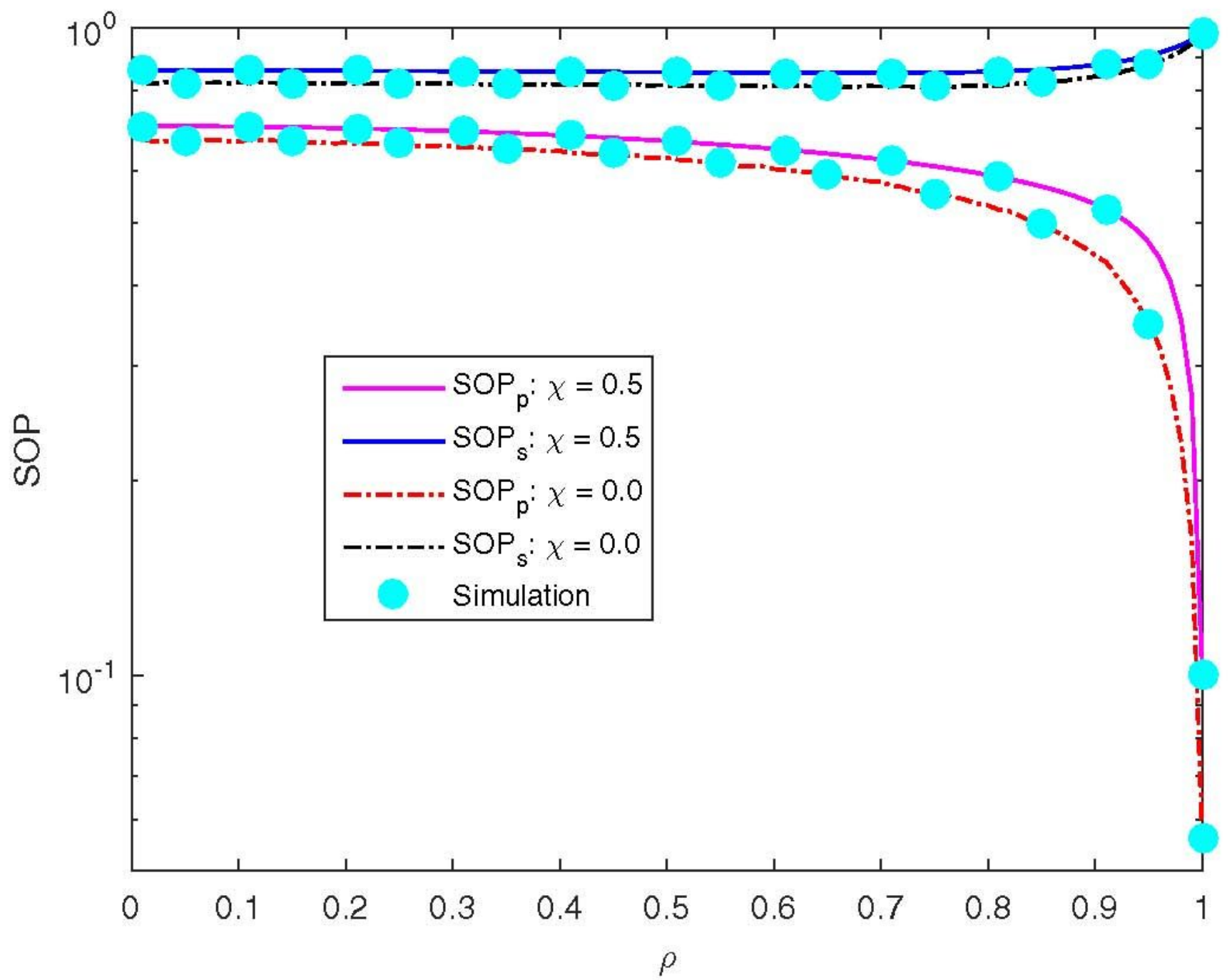

Figure 2

SOPs versus $\rho$. 


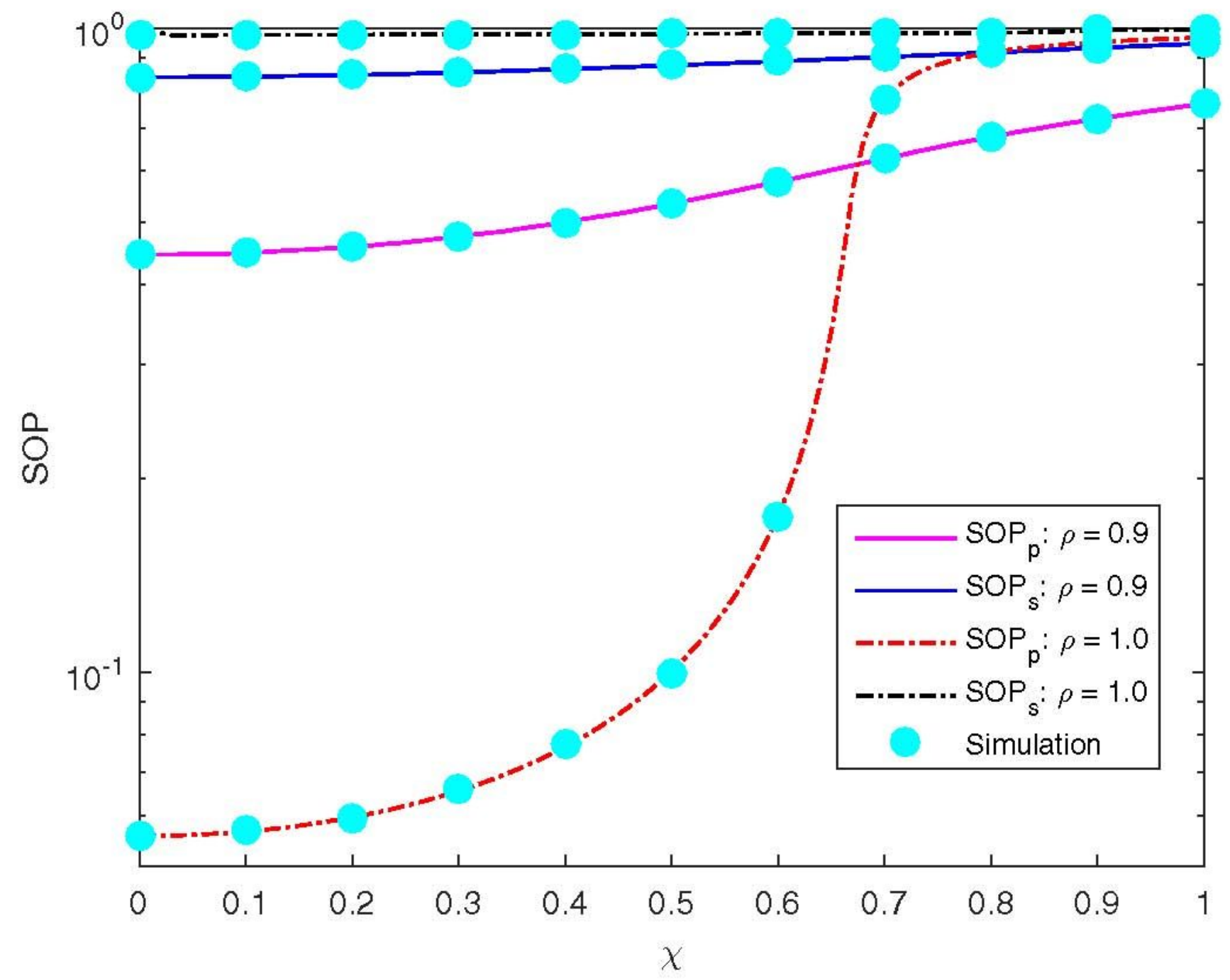

Figure 3

SOPs versus $\chi$. 


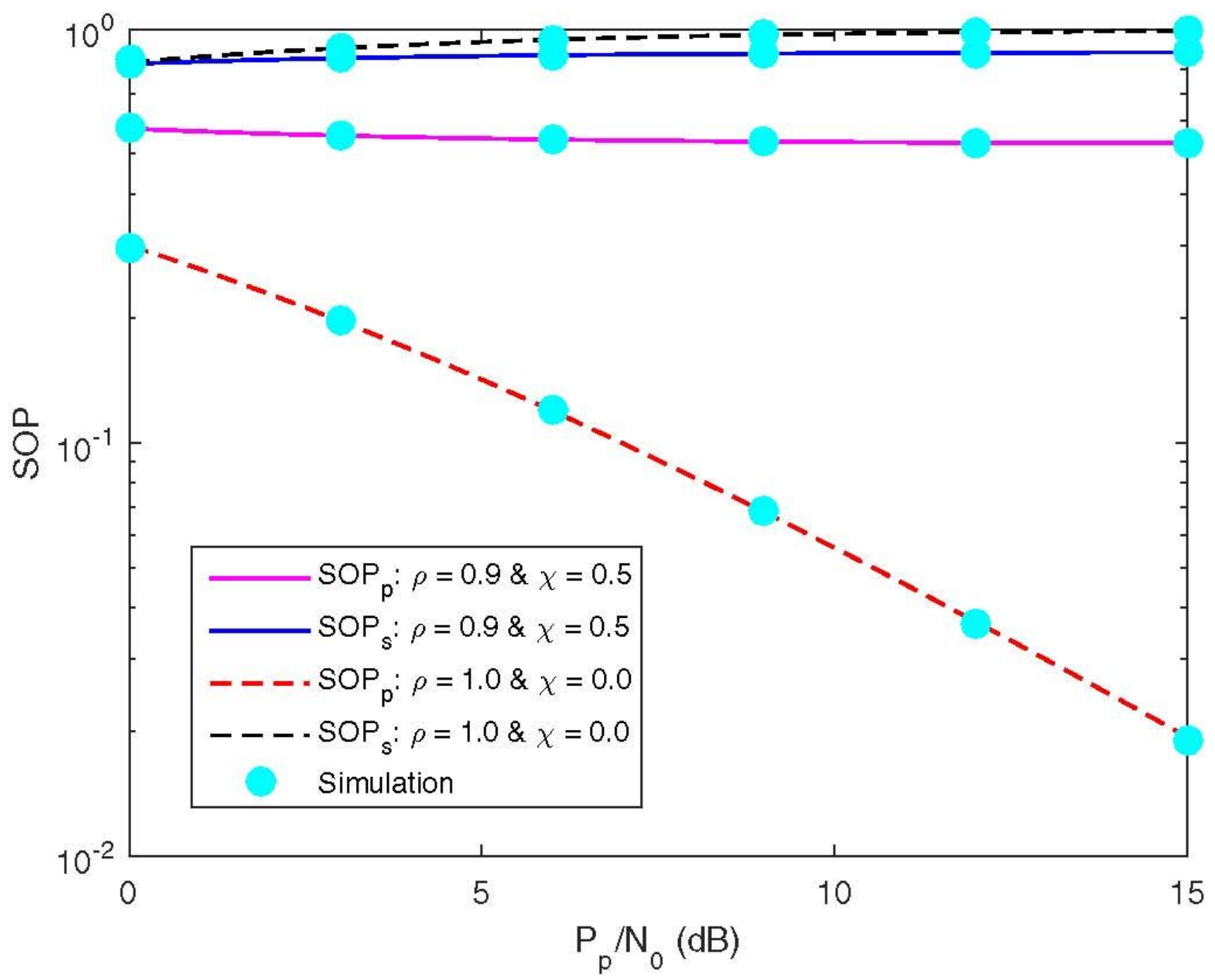

Figure 4

SOPs versus Pp/NO. 


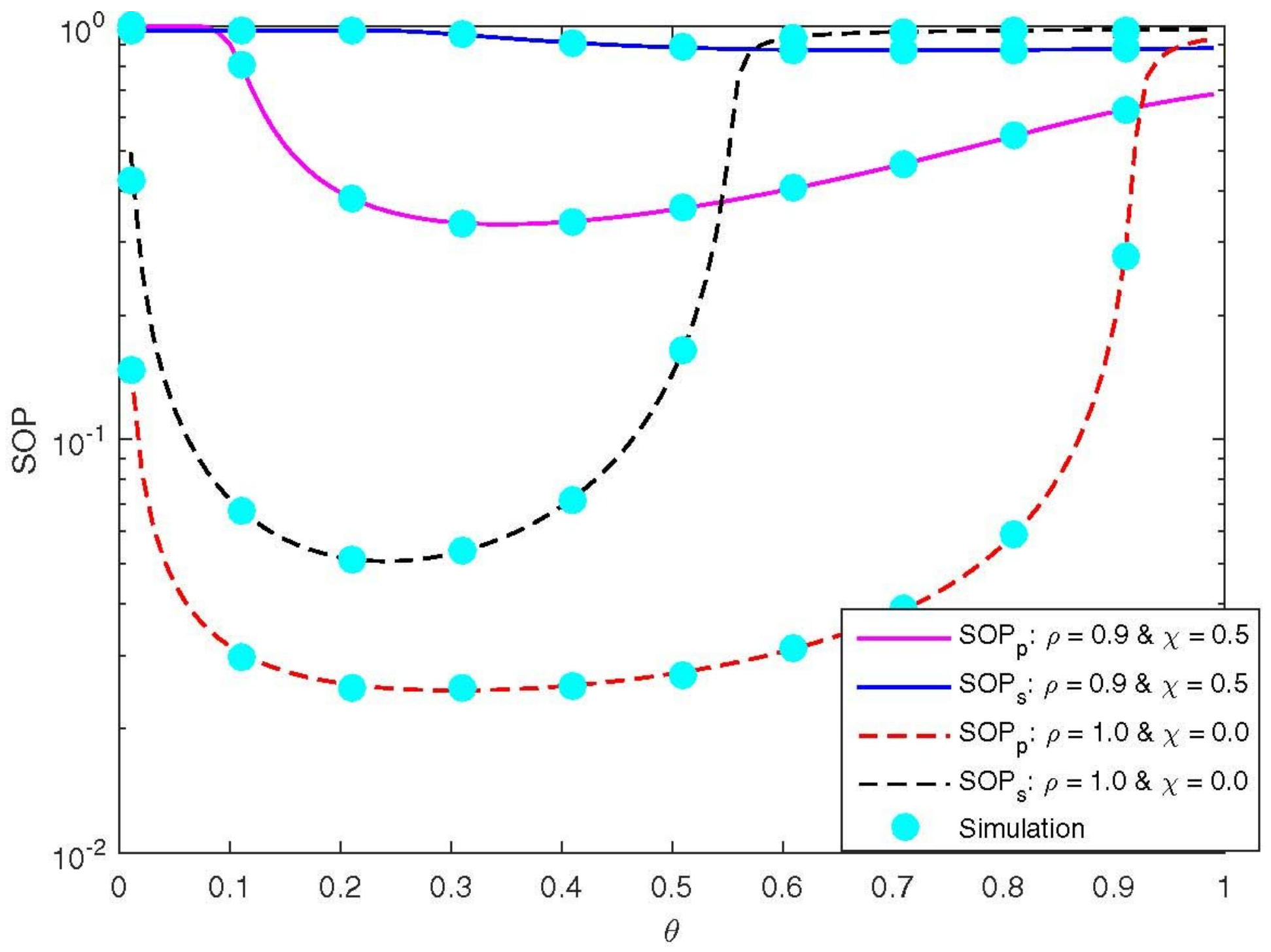

Figure 5

SOPs versus $\theta$. 


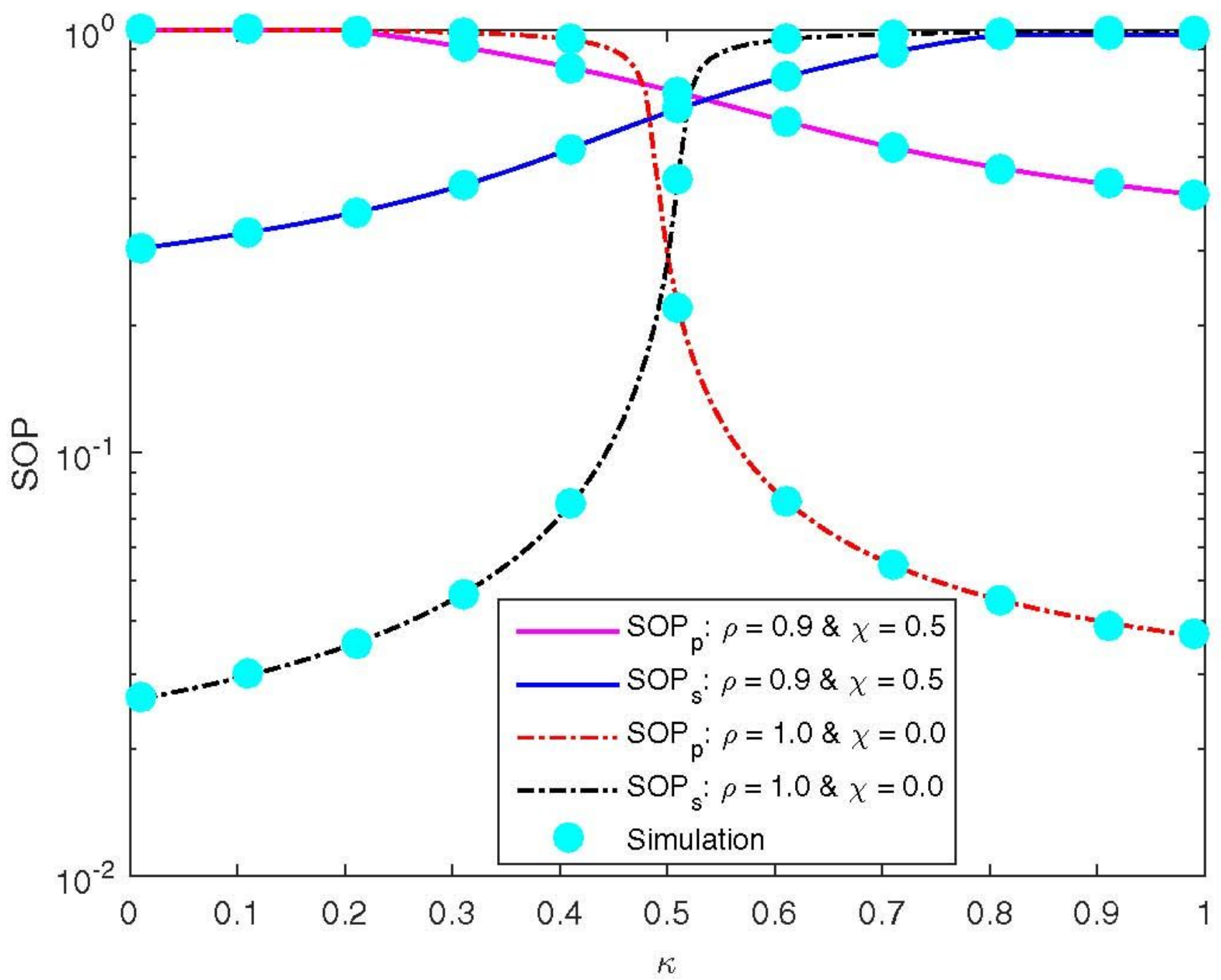

Figure 6

SOPs versus $\mathrm{K}$. 


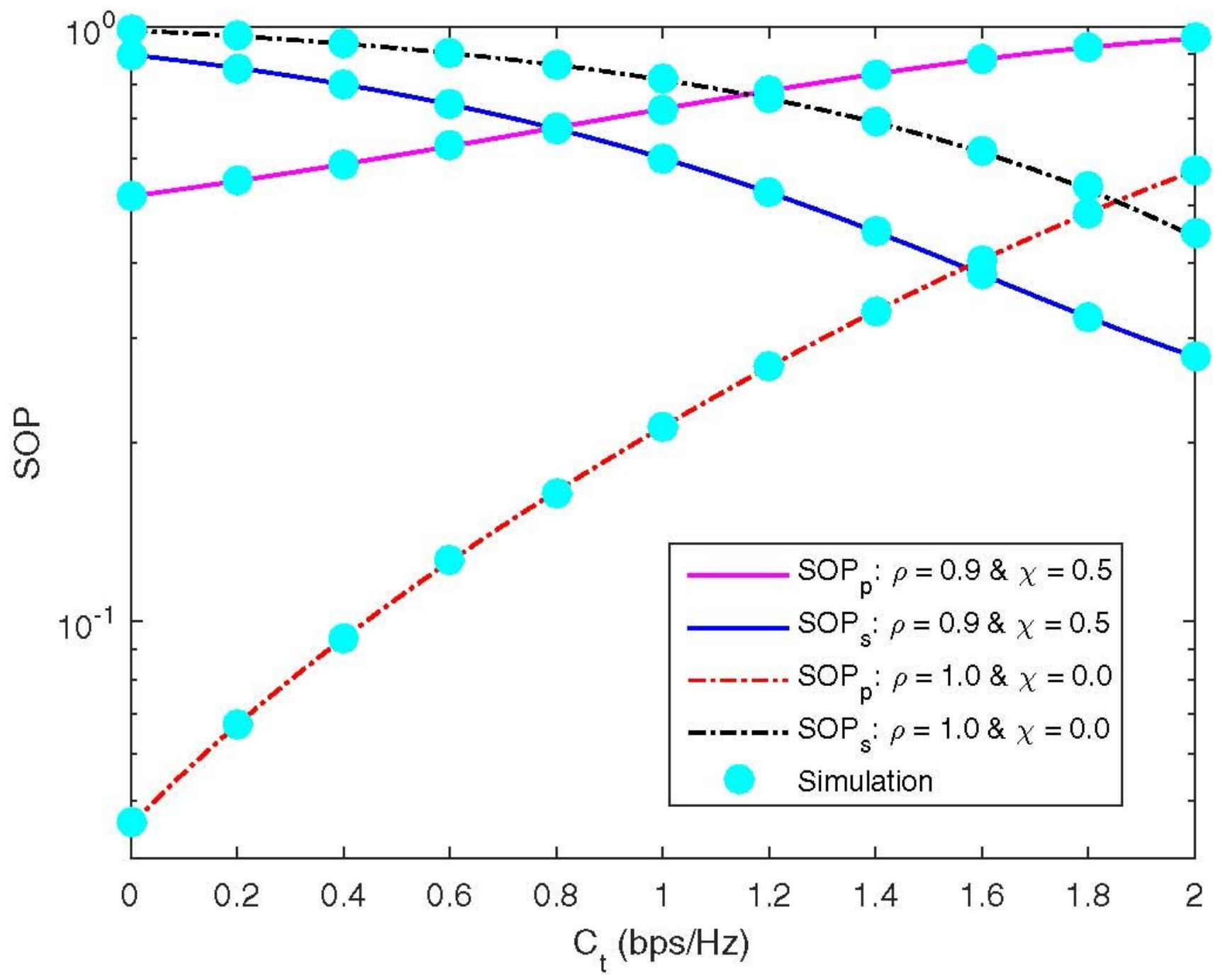

Figure 7

SOPs versus $\mathrm{Ct}$. 


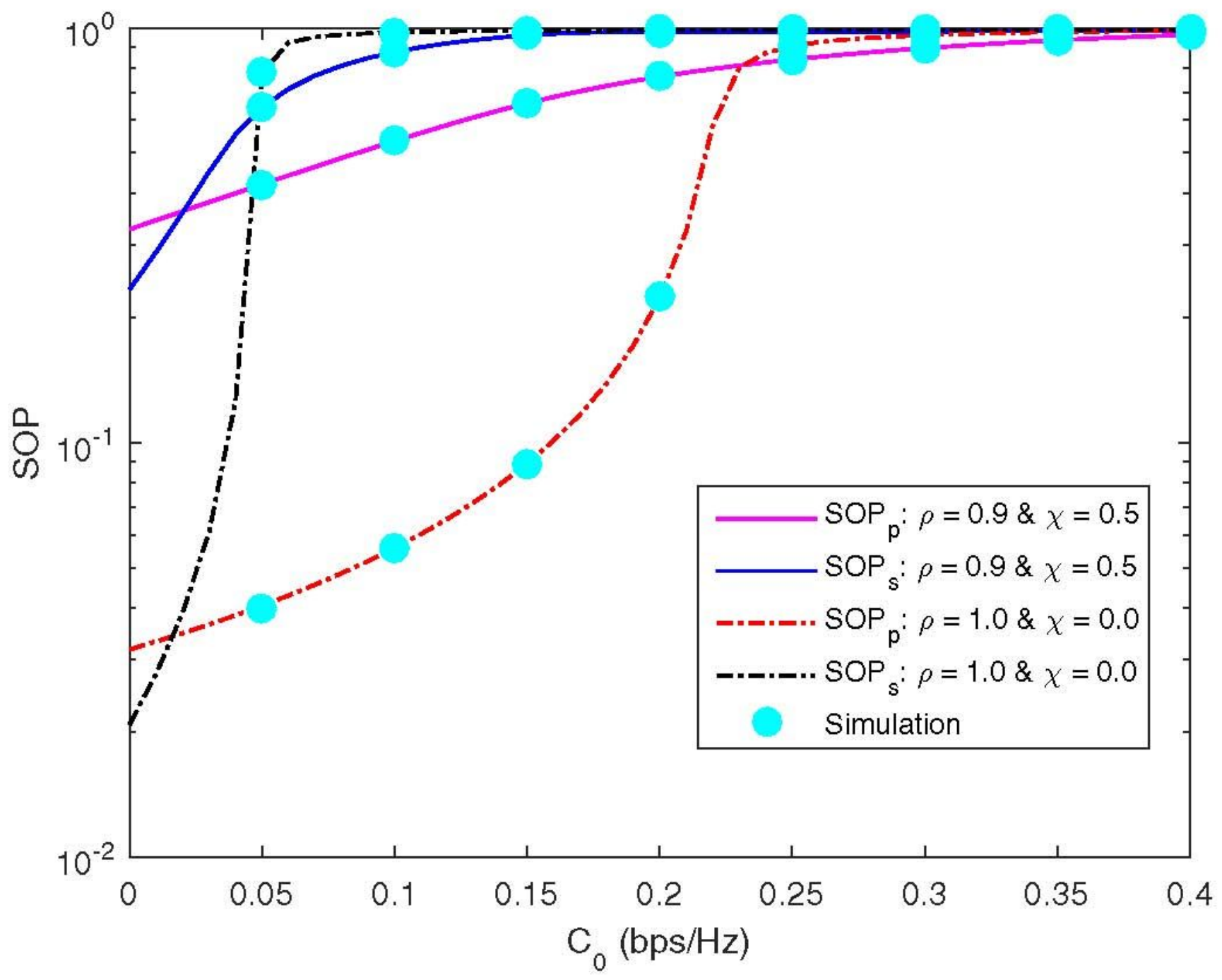

Figure 8

SOPs versus $\mathrm{CO}$. 


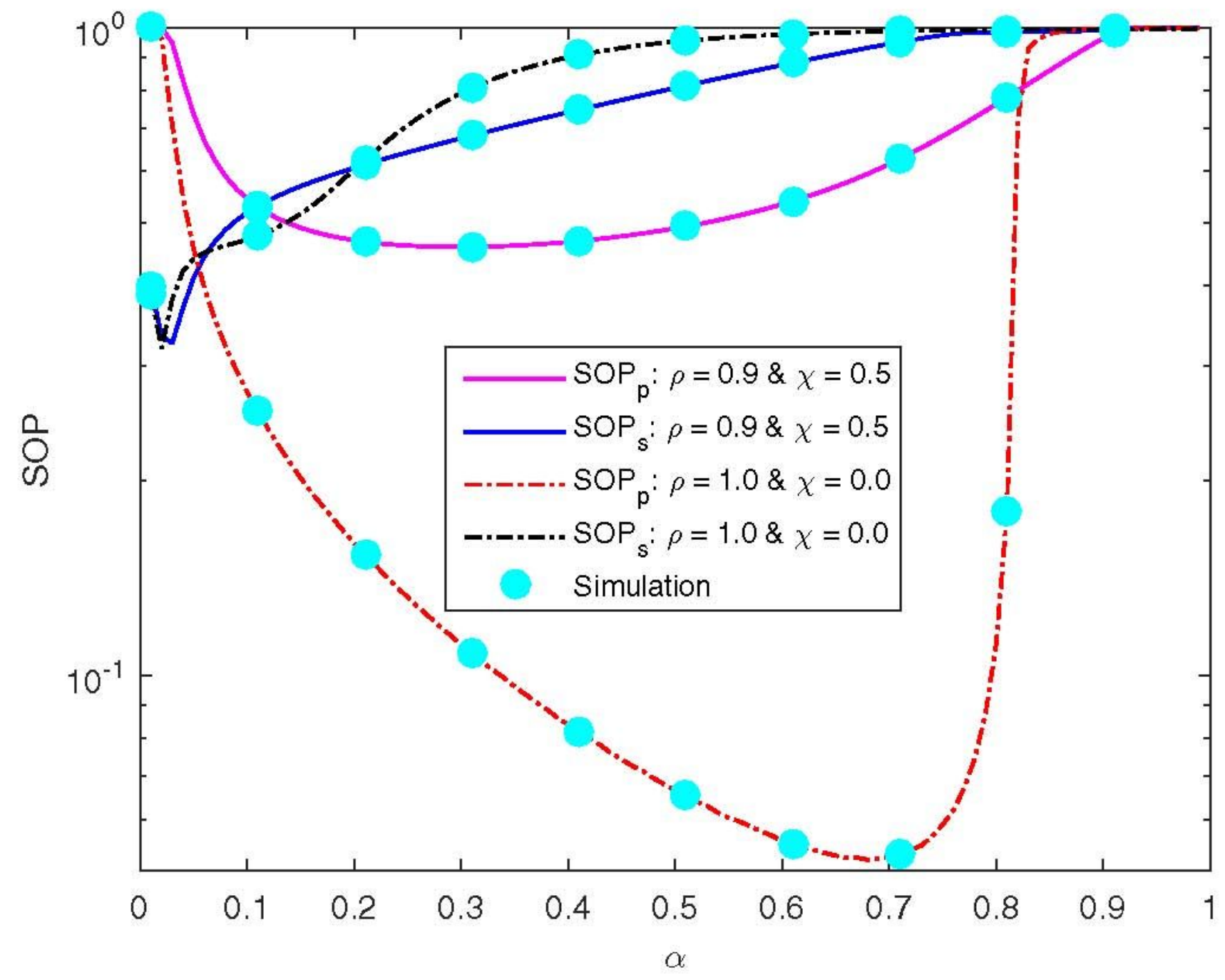

Figure 9

SOPs versus a. 


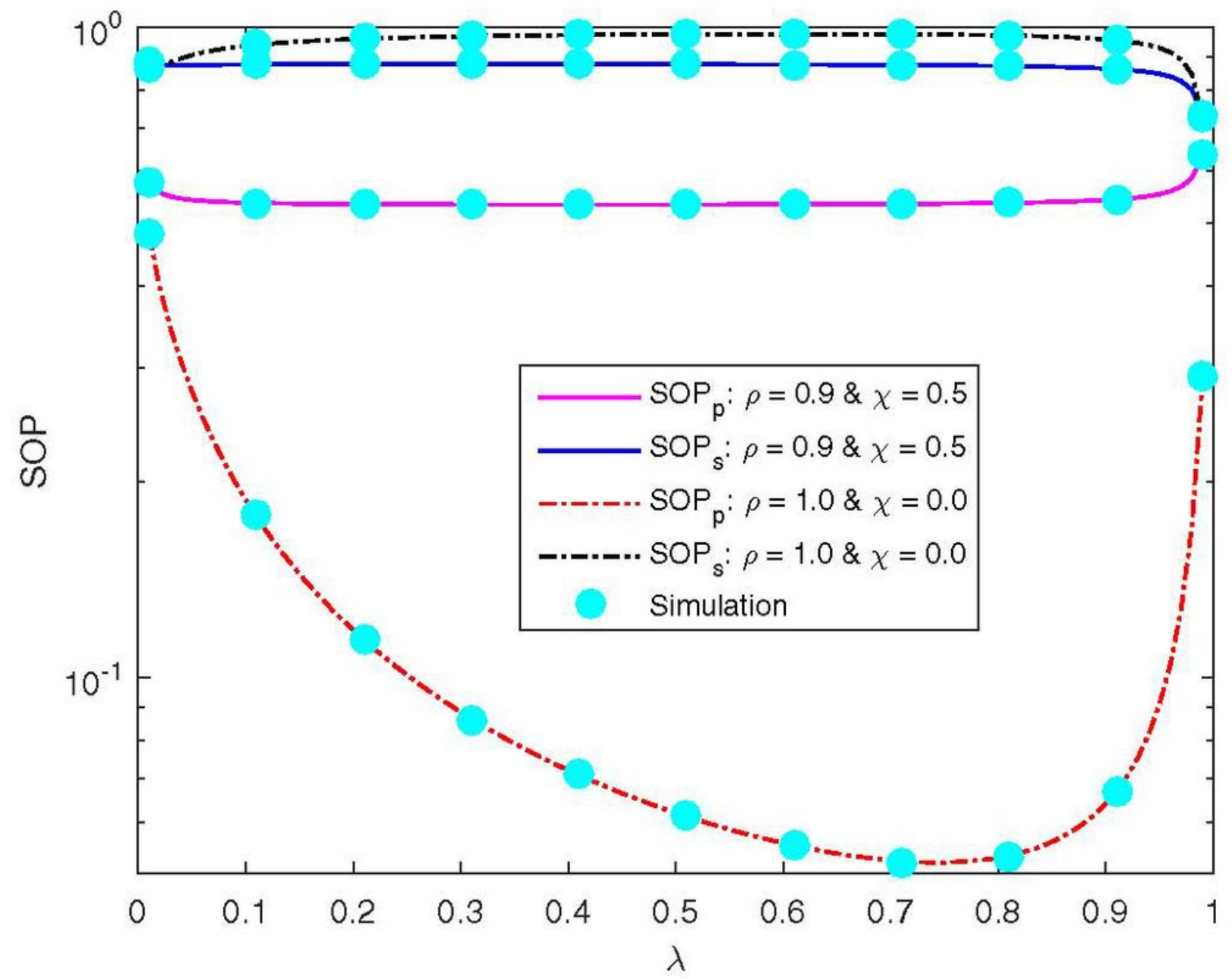

Figure 10

SOPs versus $\lambda$. 


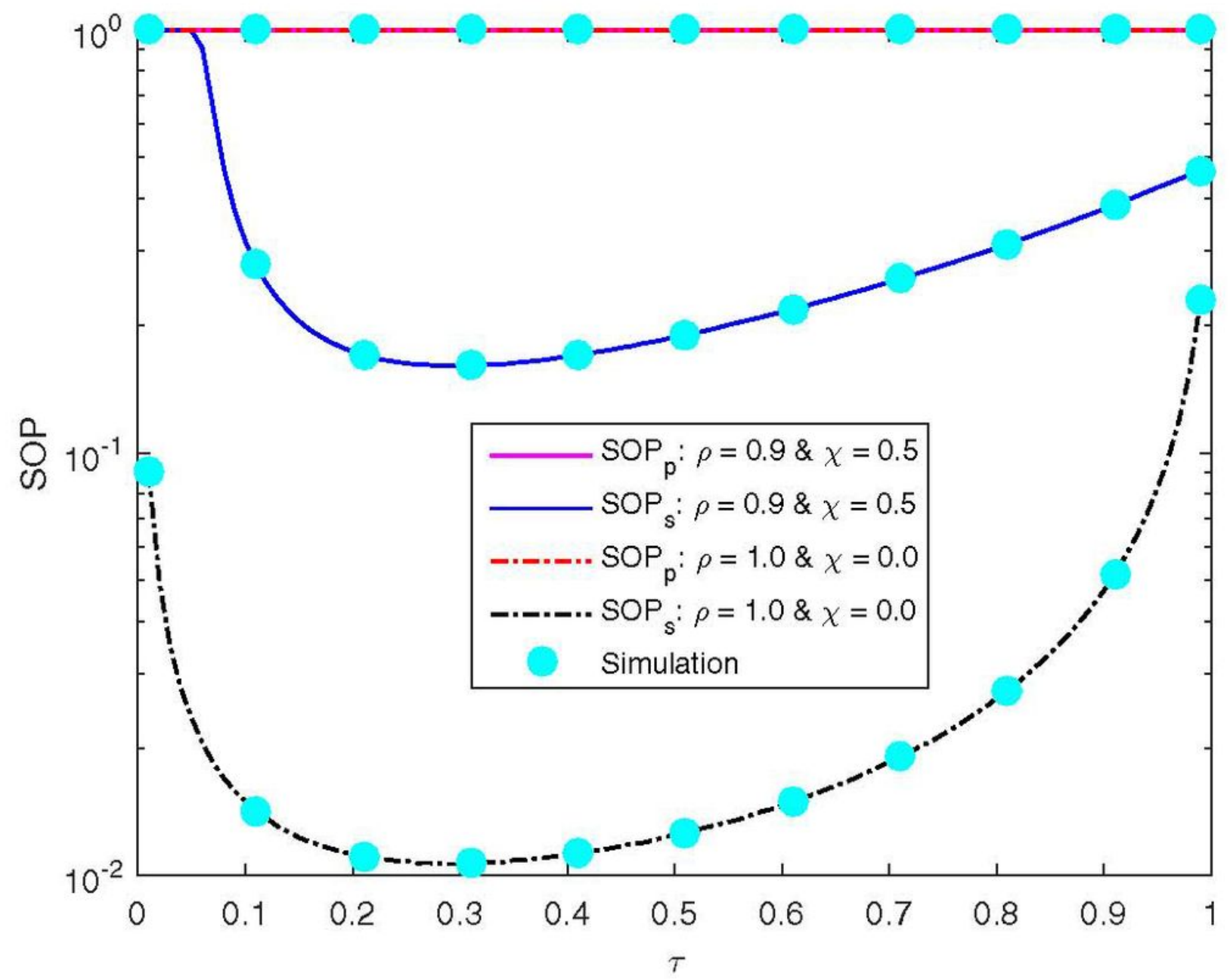

Figure 11

SOPs versus $\tau$. 

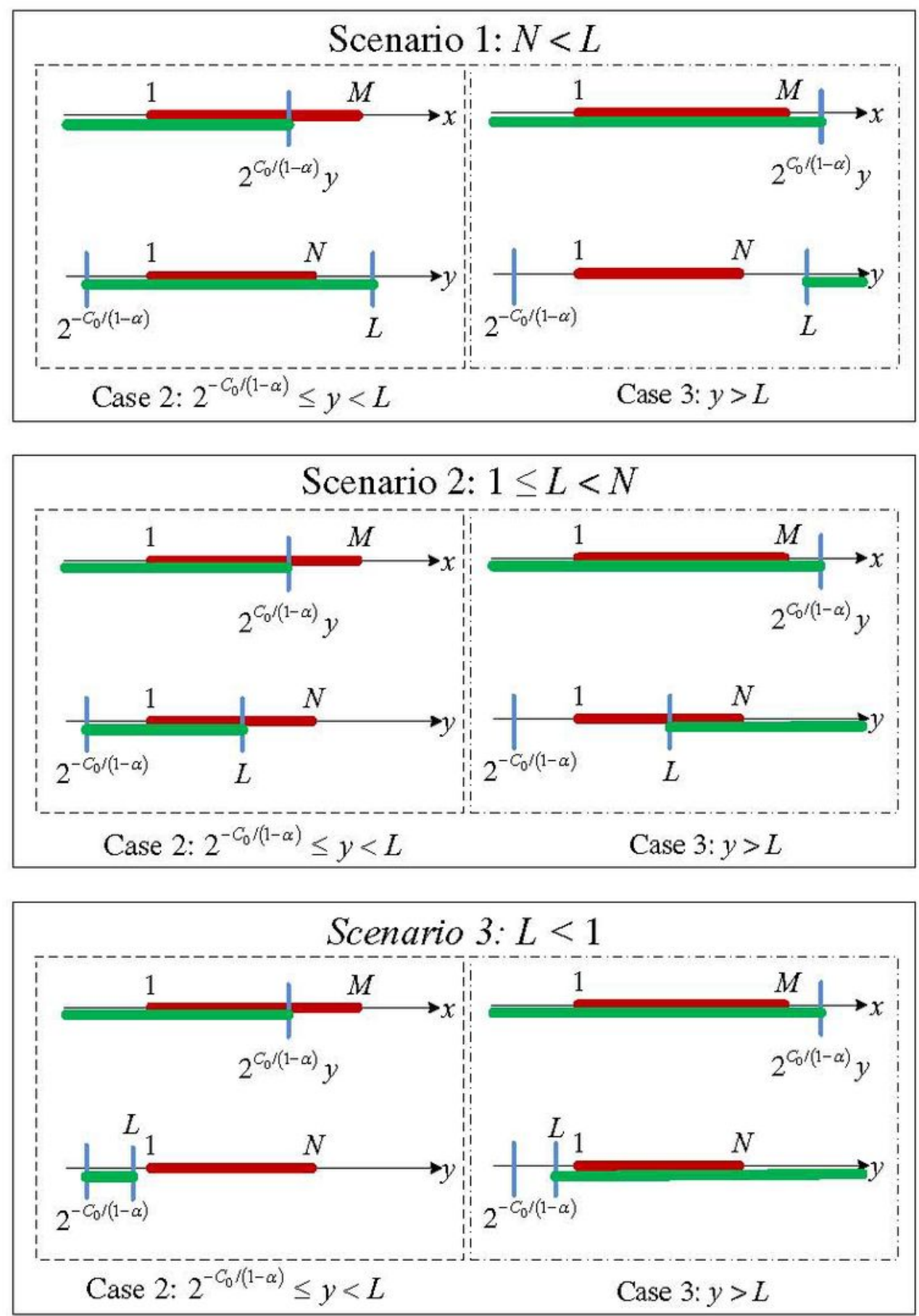

Figure 12

Scenarios for computing Y. 